\title{
Characterizations of Hemirings by the Properties of Their $k$-Ideals
}

\author{
Muhammad Shabir, Rukhshanda Anjum \\ Department of Mathematics, Quaid-i-Azam University, Islamabad, Pakistan \\ Email:mshabirbhatti@yahoo.co.uk
}

Received November 26, 2012; revised March 22, 2013; accepted March 30, 2013

Copyright (C) 2013 Muhammad Shabir, Rukhshanda Anjum. This is an open access article distributed under the Creative Commons Attribution License, which permits unrestricted use, distribution, and reproduction in any medium, provided the original work is properly cited.

\begin{abstract}
In this paper we characterize those hemirings for which each $k$-ideal is idempotent. We also characterize those hemirings for which each fuzzy $k$-ideal is idempotent. The space of prime $k$-ideals (fuzzy $k$-prime $k$-ideals) is topologized.
\end{abstract}

Keywords: Hemiring; Fuzzy k-Ideal; Idempotent k-Ideals; Prime Ideals; Semiprime Ideals

\section{Introduction}

The notion of semiring, introduced by H. S. Vandiver in 1934 [1] is a common generalization of rings and distributive lattices. Semirings play an important role in the development of automata theory, formal languages, optimization theory and other branches of applied mathematics (see for example [2-8]). Hemirings, which are semirings with commutative addition and zero element are also very important in theoretical computer science (see for instance [3,6,7]). Some other applications of semirings with references can be found in [5-7,9]. On the other hand, the notions of automata and formal languages have been generalized and extensively studied in a fuzzy frame work (cf. [8-10]).

Ideals play an important role in the structure theory of hemirings and are useful for many purposes. But they do not coincide with usual ring ideals. For this reason many results in ring theory have no analogues in semirings using only ideals. Henriksen defined in [11] a more restricted class of ideals in semirings, which is called the class of $k$-ideals. These ideals have the property that if the semiring $R$ is a ring then a subset of $R$ is a $k$-ideal if and only if it is a ring ideal. Another class of ideals is defined by Iizuka [12], which is called the class of $h$-ideals. In [13] La Torre studied these ideals, thoroughly.

The concept of fuzzy set was introduced by Zadeh in 1965 [14]. Many researchers used this concept to generalized different notions of algebra. Fuzzy semirings were first studied by Ahsan et al. [15] (see also [16]). Fuzzy $k$-ideals are studied in [17-22]. Fuzzy $h$-ideals are studied in [23-29]. In this paper we characterize those hemirnigs for which each $k$-ideal is idempotent and also those hemirings for which each fuzzy $k$-ideal is idempotent. The rest of this is organized as follows.

In Section 2, we summarize some basic concepts which will be use throughout this paper; these concepts are related to hemirings and fuzzy sets. In Section 3, $k$-product and $k$-sum of fuzzy sets in a hemiring are given. It is shown that $k$-product ( $k$-sum) of fuzzy $k$-ideals of a hemiring is a $k$-ideal. Characterization of $k$-hemiregular hemiring in terms of fuzzy left $k$-ideal and fuzzy right $k$-ideal is also given in this section. Section 4 is about idempotent fuzzy $k$-ideals of a hemiring. Different characterization of hemirings in which each fuzzy $k$-ideal is idempotent is given. In Sections 5 and 6, prime, semiprime, irreducible fuzzy $k$-ideals are studied. In last section, the space of prime $k$-ideals (fuzzy $k$-prime $k$-ideals) is topologized.

\section{Basic Results on Hemirings}

A semiring is an algebraic system $(R,+, \cdot)$ consisting of a non-empty set $R$ together with two binary operations called addition "+" and multiplication "." such that $(R,+)$ and $(R, \cdot)$ are semigroups and connecting the two algebraic structures are the distributive laws:

$$
a \cdot(b+c)=a \cdot b+a \cdot c \text { and }(b+c) \cdot a=b \cdot a+c \cdot a
$$

for all $a, b, c \in R$.

A semiring $(R,+, \cdot)$ is called a hemiring if "+" is 
commutative and $R$ has a zero element 0 , such that $a+0=0+a=a$ and $a \cdot 0=0 \cdot a=0$ for all $a \in R$. An element $1 \in R$ (if it exists) is called an identity element of $R$ if $1 \cdot a=a \cdot 1=a$ for all $a \in R$. If a hemiring contains an identity element then it is called a hemiring with identity. A hemiring $(R,+, \cdot)$ is called a commutative hemiring if ". " is commutative in $R$.

A non-empty subset $A$ of a hemiring $R$ is called a subhemiring of $R$ if $A$ itself is a hemiring with respect to the induced operations of $R$. A non-empty subset $I$ of a hemiring $R$ is called a left (right) ideal of $R$ if 1) $a+b \in I$ for all $a, b \in I$ and 2) $r a \in I \quad(a r \in I)$ for all $a \in I, r \in R$. Obviously $0 \in I$ for any left (right) ideal $I$ of $R$. A non-empty subset $I$ of a hemiring $R$ is called an ideal of $R$ if it is both a left and a right ideal of $R$. A left (right) ideal $I$ of a hemiring $R$ is called a left (right) $k$-ideal of $R$ if for any $a, b \in I$ and $x \in R$ from $x+a=b$ it follows $x \in I$.

By $k$-closure of a non-empty subset $A$ of a hemiring $R$ we mean the set

$$
\bar{A}=\{x \in R: x+a=b \text { for some } a, b \in A\} .
$$

It is clear that if $A$ is a left (right) ideal of $R$, then $A$ is the smallest left (right) $k$-ideal of $R$ containing $A$. So, $\bar{A}=A$ for all left (right) $k$-ideals of $R$. Obviously $\overline{\bar{A}}=\bar{A}$ for each non-empty $A \subseteq R$. Also $\bar{A} \subseteq \bar{B}$ for all $A \subseteq B \subseteq R$.

\subsection{Lemma}

The intersection of any family of left (right) $k$-ideals of a hemiring $R$ is a left (right) $k$-ideal of $R$.

\subsection{Lemma}

$\overline{A B}=\overline{\bar{A} \bar{B}}$ for any subsets $A, B$ of a hemiring $R$.

\subsection{Lemma}

[30] If $A$ and $B$ are, respectively, right and left $k$-ideals of a hemiring $R$, then

$$
\overline{A B} \subseteq A \cap B .
$$

\subsection{Definition}

[30] A hemiring $R$ is said to be $k$-hemiregular if for each $a \in R$, there exist $x, y \in R$ such that $a+a x a=a y a$.

\subsection{Lemma}

[30] A hemiring $R$ is $k$-hemiregular if and only if for any right $k$-ideal $A$ and any left $k$-ideal $B$, we have

$$
\overline{A B}=A \cap B .
$$

A fuzzy subset $\mu$ of a non empty set $X$ is a function $\mu: X \rightarrow[0,1] . \operatorname{Im} \mu$ denotes the set of all values of $\mu$. A fuzzy subset $\mu: X \rightarrow[0,1]$ is non-empty if there exist at least one $x \in X$ such that $\mu(x)>0$. For any fuzzy subsets $\lambda$ and $\mu$ of $X$ we define

$$
\begin{gathered}
\lambda \leq \mu \Leftrightarrow \lambda(x) \leq \mu(x), \\
(\lambda \wedge \mu)(x)=\lambda(x) \wedge \mu(x)=\min \{\lambda(x), \mu(x)\}, \\
(\lambda \vee \mu)(x)=\lambda(x) \vee \mu(x)=\max \{\lambda(x), \mu(x)\}
\end{gathered}
$$

for all $x \in X$.

More generally, if $\left\{\lambda_{i}: i \in I\right\}$ is a collection of fuzzy subsets of $X$, then by the intersection and the union of this collection we mean the fuzzy subsets

$$
\begin{aligned}
& \left(\widehat{i}_{i \in I} \lambda_{i}\right)(x)=\bigwedge_{i \in I} \lambda_{i}(x)=\inf _{i \in I}\left\{\lambda_{i}(x)\right\}, \\
& \left(\bigvee_{i \in I}^{\vee} \lambda_{i}\right)(x)=\bigvee_{i \in I}^{\vee} \lambda_{i}(x)=\sup _{i \in I}\left\{\lambda_{i}(x)\right\},
\end{aligned}
$$

respectively.

A fuzzy subset $\lambda$ of a semiring $R$ is called a fuzzy left (right) ideal of $R$ if for all $a, b \in R$ we have

1) $\lambda(a+b) \geq \lambda(a) \wedge \lambda(b)$

2) $\lambda(a b) \geq \lambda(b),(\lambda(a b) \geq \lambda(a))$.

Note that $\lambda(0) \geq \lambda(x)$ for all $x \in R$.

\subsection{Definition}

[21] A fuzzy left (right) ideal $\lambda$ of a hemiring $R$ is called a fuzzy left (right) $k$-ideal if $x+y=z \rightarrow \lambda(x) \geq \lambda(y) \wedge \lambda(z)$ for all $x, y, z \in R$.

\subsection{Definition}

Let $\lambda$ be a fuzzy subset of a universe $X$ and $t \in[0,1]$. Then the subset $U(\lambda ; t)=\{x \in X: \lambda(x) \geq t\}$ is called the level subset of $\lambda$.

\subsection{Proposition}

Let $A$ be a non-empty subset of a hemiring $R$. Then a fuzzy set $\lambda_{A}$ defined by

$$
\lambda_{A}(x)= \begin{cases}t & \text { if } x \in A \\ s & \text { otherwise }\end{cases}
$$

where $0 \leq s<t \leq 1$, is a fuzzy left (right) $k$-ideal of $R$ if and only if $A$ is a left (right) $k$-ideal of $R$.

Proof. Straightforward.

\subsection{Proposition}

[23] If $A, B$ are subsets of a hemiring $R$ such that $\operatorname{Im} \lambda_{A}=\operatorname{Im} \lambda_{B}$ then

1) $A \subseteq B \leftrightarrow \lambda_{A} \leq \lambda_{B}$,

2) $\lambda_{A} \wedge \lambda_{B}=\lambda_{A \cap B}$. 


\subsection{Proposition}

A fuzzy subset $\lambda$ of a hemiring $R$ is a fuzzy left (right) $k$-ideal of $R$ if and only if each non-empty level subset of $R$ is a left (right) $k$-ideal of $R$.

Proof. Suppose $\lambda$ is a fuzzy left $k$-ideal of $R$ and $t \in(0,1]$ such that $U(\lambda ; t) \neq \phi$. Let $a, b \in U(\lambda ; t)$, then $\lambda(a) \geq t$ and $\lambda(b) \geq t$. As $\lambda(a+b) \geq \lambda(a) \wedge \lambda(b)$, so $\lambda(a+b) \geq t$. Hence $a+b \in U(\lambda ; t)$. For $r \in R$, $\lambda(r a) \geq \lambda(a)$ so $\lambda(r a) \geq t$. This implies $r a \in U(\lambda ; t)$. Hence $U(\lambda ; t)$ is a left ideal of $R$. Now let $a+x=b$ for some $a, b \in U(\lambda ; t)$, then $\lambda(a) \geq t$ and $\lambda(b) \geq t$. Since $\lambda(x) \geq \lambda(a) \wedge \lambda(b)$, so $\lambda(x) \geq t$. Hence $x \in U(\lambda ; t)$. Thus $U(\lambda ; t)$ is a left $k$-ideal of $R$.

Conversely, assume that each non-empty subset $U(\lambda ; t)$ of $R$ is a left $k$-ideal of $R$. Let $a, b \in R$ such that $\lambda(a+b)<\lambda(a) \wedge \lambda(b)$. Take $t \in(0,1]$ such that $\lambda(a+b)<t \leq \lambda(a) \wedge \lambda(b)$, then $a, b \in U(\lambda ; t)$ but $a+b \notin U(\lambda ; t)$, a contradiction. Hence

$\lambda(a+b) \geq \lambda(a) \wedge \lambda(b)$.

Similarly we can show that $\lambda(a b) \geq \lambda(b)$.

Let $x, y, z \in R$ such that $x+y=z$. If possible let $\lambda(x)<\lambda(y) \wedge \lambda(z)$. Take $t \in(0,1]$ such that $\lambda(x)<$ $t \leq \lambda(y) \wedge \lambda(z)$, then $y, z \in U(\lambda ; t)$ but $x \notin U(\lambda ; t)$, a contradiction. Hence $\lambda(x) \geq \lambda(y) \wedge \lambda(z)$. Thus $\lambda$ is a fuzzy left $k$-ideal of $R$.

\subsection{Example}

The set $R=\{0,1,2,3\}$ with operations addition and multiplication given by the following Cayley tables:

\begin{tabular}{ccccc}
\hline+ & 0 & 1 & 2 & 3 \\
\hline 0 & 0 & 1 & 2 & 3 \\
1 & 1 & 1 & 2 & 3 \\
2 & 2 & 2 & 2 & 3 \\
3 & 3 & 3 & 3 & 2 \\
\hline$\cdot$ & 0 & 1 & 2 & 3 \\
\hline 0 & 0 & 0 & 0 & 0 \\
1 & 0 & 1 & 1 & 1 \\
2 & 0 & 1 & 1 & 1 \\
3 & 0 & 1 & 1 & 1 \\
\hline
\end{tabular}

is a hemiring. Ideals in $R$ are $\{0\},\{0,1\},\{0,1,2\}$, $\{0,1,2,3\}$. All ideals are $k$-ideals. Let $t_{1}, t_{2}, t_{3}, t_{4} \in(0,1]$ such that $t_{1} \geq t_{2} \geq t_{3} \geq t_{4}$.

Define $\lambda: R \rightarrow[0,1]$ by

$$
\lambda(0)=t_{1}
$$

$$
\begin{aligned}
& \lambda(1)=t_{2} \\
& \lambda(2)=t_{3} \\
& \lambda(3)=t_{4}
\end{aligned}
$$

Then

$$
U(\lambda ; t)= \begin{cases}\{0,1,2,3\} & \text { if } t \leq t_{4} \\ \{0,1,2\} & \text { if } t_{4}<t \leq t_{3} \\ \{0,1\} & \text { if } t_{3}<t \leq t_{2} \\ \{0\} & \text { if } t_{2}<t \leq t_{1} \\ \phi & \text { if } t>t_{1}\end{cases}
$$

Thus by Proposition 2.10, $\lambda$ is a fuzzy $k$-ideal of $R$.

\section{3. $\boldsymbol{k}$-Product of Fuzzy Subsets}

To avoid repetitions from now $R$ will always mean a hemiring $(R,+, \cdot)$.

\subsection{Definition}

The $k$-product of two fuzzy subsets $\mu$ and $v$ of $R$ is defined by

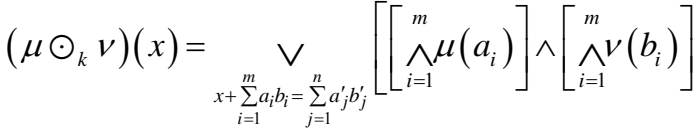

$$
\begin{aligned}
& \left.\wedge\left[{ }_{j=1}^{n} \mu\left(a_{j}^{\prime}\right)\right] \wedge\left[{ }_{j=1}^{n} v\left(b_{j}^{\prime}\right)\right]\right]
\end{aligned}
$$

and $\left(\mu \odot_{k} v\right)(x)=0$ if $x$ can not be expressed as

$$
x+\sum_{i=1}^{m} a_{i} b_{i}=\sum_{j=1}^{n} a_{j}^{\prime} b_{j}^{\prime} .
$$

By direct calculations we obtain the following result.

\subsection{Proposition}

Let $\mu, v, \omega, \lambda$ be fuzzy subsets of $R$. Then $\mu \leq \omega$ and $v \leq \lambda \rightarrow \mu \odot_{k} v \leq \omega \odot_{k} \lambda$.

For any subset $A$ in a hemiring $R, \chi_{\mathrm{A}}$ will denote the characteristic function of $A$.

\subsection{Lemma}

Let $R$ be a hemiring and $A, B \subseteq R$. Then we have

1) $A \subseteq B$ if and only if $\chi_{A} \subseteq \chi_{B}$.

2) $\chi_{A} \cap \chi_{B}=\chi_{A \cap B}$.

3) $\chi_{A} \odot_{k} \chi_{B}=\chi_{\overline{A B}}$.

Proof. 1) and 2) are obvious. For 3) let $x \in R$. If $x \in \overline{A B}$, then $\chi_{\overline{A B}}(x)=1$ and $x+\sum_{i=1}^{m} p_{i} q_{i}=\sum_{j=1}^{n} p_{j}^{\prime} q_{j}^{\prime}$ for some $p_{i}, p_{j}^{\prime} \in A$ and $q_{i}, q_{j}^{\prime} \in B$. Thus we have 
and so

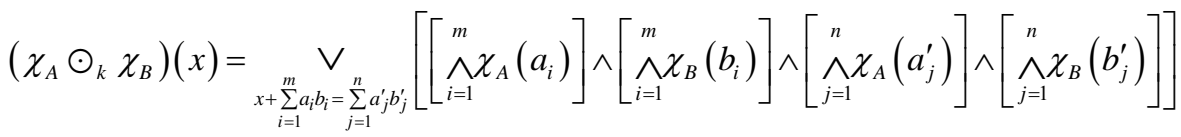

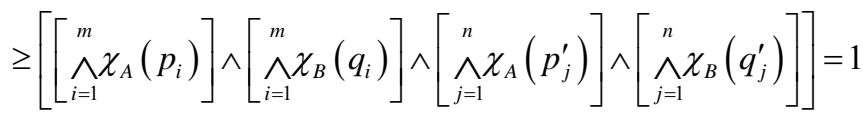

$$
\left(\chi_{A} \odot_{k} \chi_{B}\right)(x)=1=\chi_{\overline{A B}}
$$

If $x \notin \overline{A B}$, then $\chi_{\overline{A B}}=0$. If possible, let $\left(\chi_{A} \odot_{k} \chi_{B}\right)(x) \neq 0$. Then

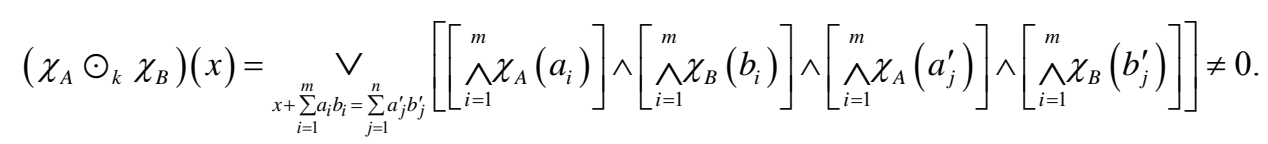

Hence there exist $p_{i}, q_{i}, p_{j}^{\prime}, q_{j}^{\prime} \in R$ such that

$$
x+\sum_{i=1}^{m} p_{i} q_{i}=\sum_{j=1}^{n} p_{j}^{\prime} q_{j}^{\prime}
$$

and

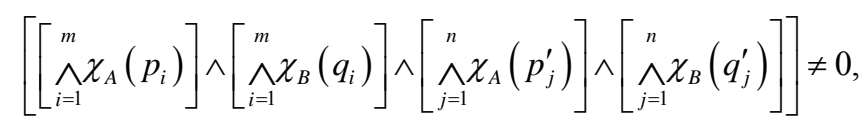

that is

$$
\chi_{A}\left(p_{i}\right)=\chi_{A}\left(p_{j}^{\prime}\right)=\chi_{B}\left(q_{i}\right)=\chi_{B}\left(q_{j}^{\prime}\right)=1,
$$

hence $p_{i}, p_{j}^{\prime} \in A$ and $q_{i}, q_{j}^{\prime} \in B$, and so $x \in \overline{A B}$ which is a contradiction. Thus we have

$$
\left(\chi_{A} \odot_{k} \chi_{B}\right)(x)=0=\chi_{\overline{A B}}(x) .
$$

Hence in any case, we have

$$
\left(\chi_{A} \odot_{k} \chi_{B}\right)(x)=\chi_{\overline{A B}}(x) .
$$

\subsection{Theorem}

If $\lambda, \mu$ are fuzzy $k$-ideals of $R$, then $\lambda \odot_{k} \mu$ is a fuzzy $k$-ideal of $R$ and $\lambda \odot_{k} \mu \leq \lambda \wedge \mu$.

Proof. Let $\lambda, \mu$ be fuzzy $k$-ideals of $R$. Let $x, y \in R$, then

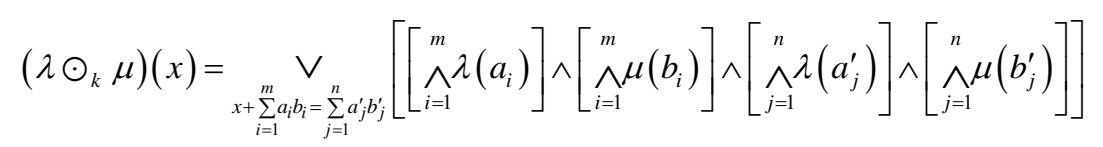

and

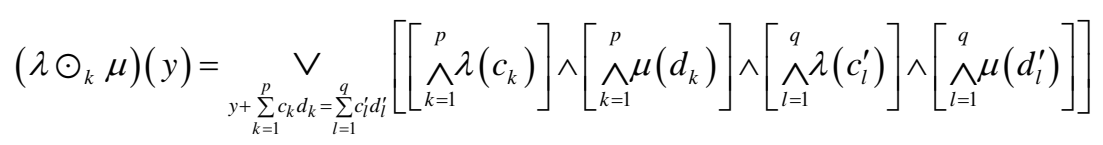

Thus

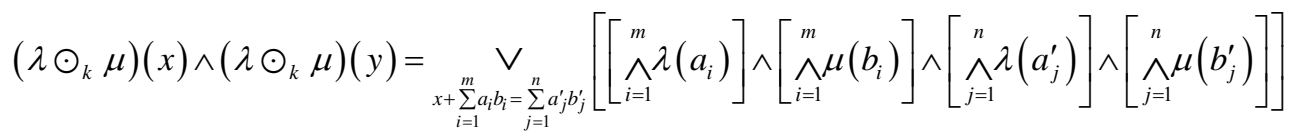

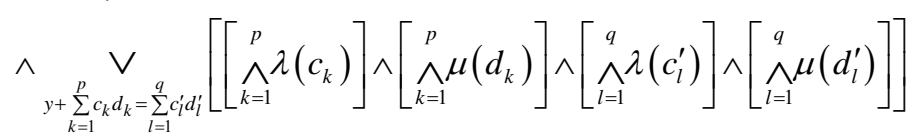

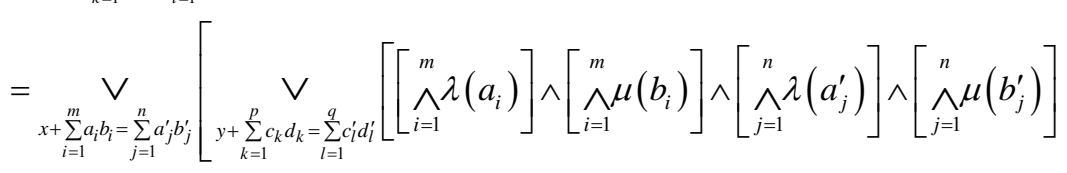

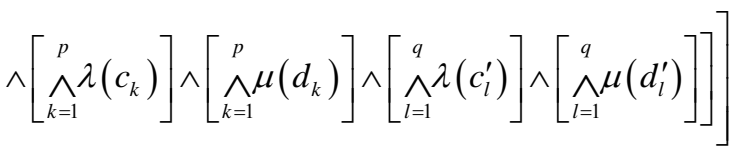


Since for each expression $x+\sum_{i=1}^{m} a_{i} b_{i}=\sum_{j=1}^{n} a_{j}^{\prime} b_{j}^{\prime}$ and $y+\sum_{k=1}^{p} c_{k} d_{k}=\sum_{l=1}^{q} c_{l}^{\prime} d_{l}^{\prime} \quad$ we have

so we have

$$
x+y+\sum_{i=1}^{m} a_{i} b_{i}+\sum_{k=1}^{p} c_{k} d_{k}=\sum_{l=1}^{q} c_{l}^{\prime} d_{l}^{\prime}+\sum_{j=1}^{n} a_{j}^{\prime} b_{j}^{\prime},
$$

$$
\left(\lambda \odot_{k} \mu\right)(x) \wedge\left(\lambda \odot_{k} \mu\right)(y) \leq \underset{x+y+\sum_{s=1}^{u} e_{s} f_{s}=\sum_{t=1}^{v} e_{f}^{\prime} f_{t}^{\prime}}{\vee}\left[\left[\bigwedge_{s=1}^{u} \lambda\left(e_{s}\right)\right] \wedge\left[\bigwedge_{s=1}^{u} \mu\left(f_{s}\right)\right] \wedge\left[\bigwedge_{t=1}^{v} \lambda\left(e_{t}^{\prime}\right)\right] \wedge\left[\bigwedge_{t=1}^{v} \mu\left(f_{t}^{\prime}\right)\right]\right]=\left(\lambda \odot_{k} \mu\right)(x+y)
$$

Similarly,

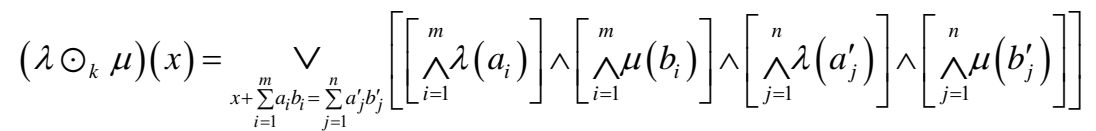

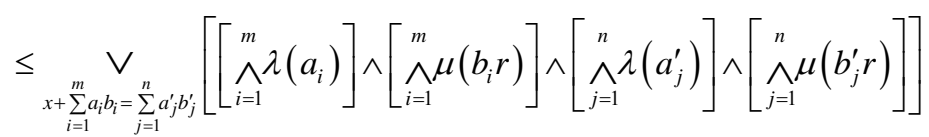

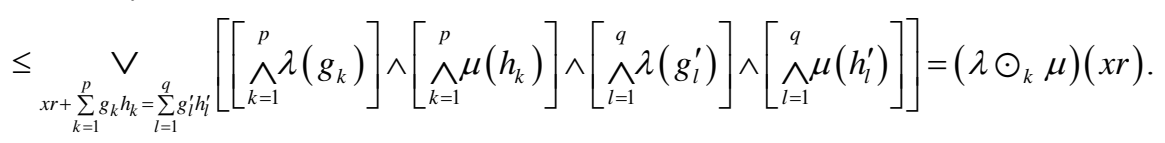

Analogously we can verify that $\left(\lambda \odot_{k} \mu\right)(x r) \geq\left(\lambda \odot_{k} \mu\right)(x)$ for all $r, x \in R$. This means that $\lambda \odot_{k} \mu$ is a fuzzy ideal of $R$.

To prove that $x+a=b$ implies

$$
\left(\lambda \odot_{k} \mu\right)(x) \geq\left(\lambda \odot_{k} \mu\right)(a) \wedge\left(\lambda \odot_{k} \mu\right)(b)
$$

observe that

$$
a+\sum_{i=1}^{m} a_{i} b_{i}=\sum_{j=1}^{n} a_{j}^{\prime} b_{j}^{\prime} \text { and } b+\sum_{k=1}^{l} c_{k} d_{k}=\sum_{q=1}^{p} c_{q}^{\prime} d_{q}^{\prime},
$$

together with $x+a=b$, gives $x+a+\sum_{i=1}^{m} a_{i} b_{i}=b+\sum_{i=1}^{m} a_{i} b_{i}$. Thus

$$
x+\sum_{j=1}^{n} a_{j}^{\prime} b_{j}^{\prime}=b+\sum_{i=1}^{m} a_{i} b_{i}
$$

and, consequently,

$$
\begin{aligned}
& x+\sum_{j=1}^{n} a_{j}^{\prime} b_{j}^{\prime}+\sum_{k=1}^{l} c_{k} d_{k}=b+\sum_{k=1}^{l} c_{k} d_{k}+\sum_{i=1}^{m} a_{i} b_{i} \\
& =\sum_{q=1}^{p} c_{q}^{\prime} d_{q}^{\prime}+\sum_{i=1}^{m} a_{i} b_{i}=\sum_{i=1}^{m} a_{i} b_{i}+\sum_{q=1}^{p} c_{q}^{\prime} d_{q}^{\prime}
\end{aligned}
$$

Therefore

$$
x+\sum_{j=1}^{n} a_{j}^{\prime} b_{j}^{\prime}+\sum_{k=1}^{l} c_{k} d_{k}=\sum_{i=1}^{m} a_{i} b_{i}+\sum_{q=1}^{p} c_{q}^{\prime} d_{q}^{\prime} .
$$

Now, we have

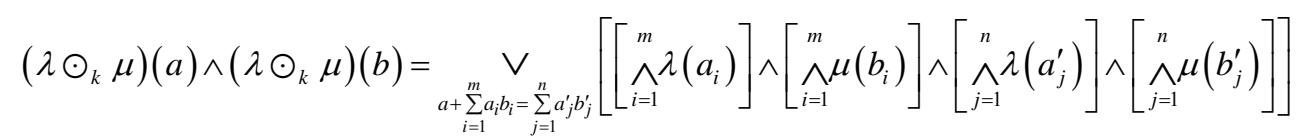

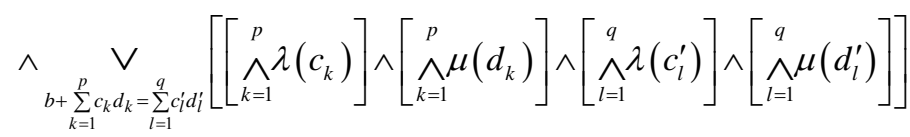

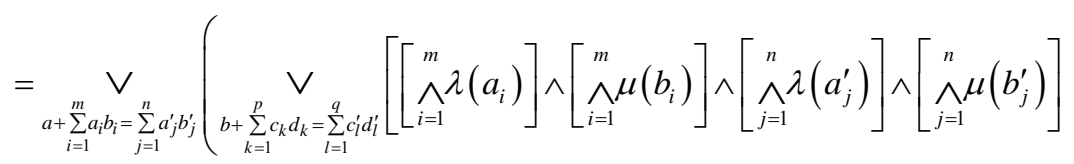

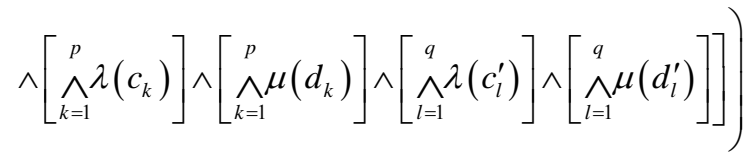

$$
\begin{aligned}
& \leq \underset{x+\sum_{s=1}^{u} g_{s} h_{s}=\sum_{t=1}^{w} g_{t}^{\prime} h_{t}^{\prime}}{ }\left(\left[\bigwedge_{s=1}^{u} \lambda\left(g_{s}\right)\right] \wedge\left[\bigwedge_{s=1}^{u} \mu\left(h_{s}\right)\right] \wedge\left[\bigwedge_{t=1}^{w} \lambda\left(g_{t}^{\prime}\right)\right] \wedge\left[\bigwedge_{t=1}^{w} \mu\left(h_{t}^{\prime}\right)\right]\right)=\left(\lambda \odot_{k} \mu\right)(x) .
\end{aligned}
$$


Thus

$$
\left(\lambda \odot_{k} \mu\right)(a) \wedge\left(\lambda \odot_{k} \mu\right)(b) \leq\left(\lambda \odot_{k} \mu\right)(x) .
$$

Hence $\left(\lambda \odot_{k} \mu\right)$ is a fuzzy $k$-ideal of $R$.

By simple calculations we can prove that

$$
\lambda \odot_{k} \mu \leq \lambda \wedge \mu .
$$

\subsection{Definition}

The $k$-sum $\lambda+{ }_{k} \mu$ of fuzzy subsets $\lambda$ and $\mu$ of $R$ is defined by

$$
\begin{aligned}
& \left(\lambda+{ }_{k} \mu\right)(x) \\
& =\sup _{x+\left(a_{1}+b_{1}\right)=\left(a_{2}+b_{2}\right)}\left[\lambda\left(a_{1}\right) \wedge \lambda\left(a_{2}\right) \wedge \mu\left(b_{1}\right) \wedge \mu\left(b_{2}\right)\right],
\end{aligned}
$$

where $x, a_{1}, b_{1}, a_{2}, b_{2} \in R$.

\subsection{Theorem}

The $k$-sum of fuzzy $k$-ideals of $R$ is also a fuzzy $k$-ideal of $R$.

Proof. Let $\lambda, \mu$ be fuzzy $k$-ideals of $R$. Then for $x, y, r \in R$ we have

$$
\begin{aligned}
& \left(\lambda+{ }_{k} \mu\right)(x) \wedge\left(\lambda+{ }_{k} \mu\right)(y) \\
& =\left[\underset{x+\left(a_{1}+b_{1}\right)=\left(a_{2}+b_{2}\right)}{\vee}\left[\lambda\left(a_{1}\right) \wedge \lambda\left(a_{2}\right) \wedge \mu\left(b_{1}\right) \wedge \mu\left(b_{2}\right)\right]\right] \wedge\left[\underset{x+\left(a_{1}^{\prime}+b_{1}^{\prime}\right)=\left(a_{2}^{\prime}+b_{2}^{\prime}\right)}{\vee}\left[\lambda\left(a_{1}^{\prime}\right) \wedge \lambda\left(a_{2}^{\prime}\right) \wedge \mu\left(b_{1}^{\prime}\right) \wedge \mu\left(b_{2}^{\prime}\right)\right]\right] \\
& =\underset{\begin{array}{c}
x+\left(a_{1}+b_{1}\right)=\left(a_{2}+b_{2}\right) \\
x+\left(a_{1}^{\prime}+b_{1}^{\prime}\right)=\left(a_{2}^{\prime}+b_{2}^{\prime}\right)
\end{array}}{\vee}\left(\lambda\left(a_{1}\right) \wedge \lambda\left(a_{2}\right) \wedge \mu\left(b_{1}\right) \wedge \mu\left(b_{2}\right) \wedge \lambda\left(a_{1}^{\prime}\right) \wedge \lambda\left(a_{2}^{\prime}\right) \wedge \mu\left(b_{1}^{\prime}\right) \wedge \mu\left(b_{2}^{\prime}\right)\right) \\
& =\underset{\substack{x+\left(a_{1}+b_{1}\right)=\left(a_{2}+b_{2}\right) \\
x+\left(a_{1}^{\prime}+b_{1}^{\prime}\right)=\left(a_{2}^{\prime}+b_{2}^{\prime}\right)}}{ }\left(\lambda\left(a_{1}+a_{1}^{\prime}\right) \wedge \lambda\left(a_{2}+a_{2}^{\prime}\right) \wedge \mu\left(b_{1}+b_{1}^{\prime}\right) \wedge \mu\left(b_{2}+b_{2}^{\prime}\right)\right) \\
& \leq \underset{(x+y)+\left(c_{1}+d_{1}\right)=\left(c_{2}+d_{2}\right)}{\vee}\left[\lambda\left(c_{1}\right) \wedge \lambda\left(c_{2}\right) \wedge \mu\left(d_{1}\right) \wedge \mu\left(d_{2}\right)\right]=\left(\lambda+{ }_{k} \mu\right)(x+y) .
\end{aligned}
$$

Similarly,

$$
\begin{aligned}
\left(\lambda+{ }_{k} \mu\right)(x) & =\underset{x+\left(a_{1}+b_{1}\right)=\left(a_{2}+b_{2}\right)}{\vee}\left[\lambda\left(a_{1}\right) \wedge \lambda\left(a_{2}\right) \wedge \mu\left(b_{1}\right) \wedge \mu\left(b_{2}\right)\right] \leq \underset{x+\left(a_{1}+b_{1}\right)=\left(a_{2}+b_{2}\right)}{\vee}\left[\lambda\left(r a_{1}\right) \wedge \lambda\left(r a_{2}\right) \wedge \mu\left(r b_{1}\right) \wedge \mu\left(r b_{2}\right)\right] \\
& \leq V_{r x+\left(a_{1}^{\prime \prime}+b_{1}^{\prime \prime}\right)=\left(a_{2}^{\prime \prime}+b_{2}^{\prime \prime}\right)}\left[\lambda\left(a_{1}^{\prime \prime}\right) \wedge \lambda\left(a_{2}^{\prime \prime}\right) \wedge \mu\left(b_{1}^{\prime \prime}\right) \wedge \mu\left(b_{2}^{\prime \prime}\right)\right]=\left(\lambda+{ }_{k} \mu\right)(r x) .
\end{aligned}
$$

Similarly $\left(\lambda+_{k} \mu\right)(x) \leq\left(\lambda+_{k} \mu\right)(r x)$. This proves and that $\left(\lambda+{ }_{k} \mu\right)$ is a fuzzy ideal of $R$.

Now we show that $x+a=b$ implies $\left(\lambda+{ }_{k} \mu\right)(x) \geq\left(\lambda+{ }_{k} \mu\right)(a) \wedge\left(\lambda+{ }_{k} \mu\right)(b)$. For this let $a+\left(a_{1}+b_{1}\right)=\left(a_{2}+b_{2}\right)$ and $b+\left(c_{1}+d_{1}\right)=\left(c_{2}+d_{2}\right)$ Then,

$$
x+a+\left(c_{1}+d_{1}\right)=\left(c_{2}+d_{2}\right)
$$

whence

$$
\begin{aligned}
& x+a+\left(c_{1}+d_{1}\right)+\left(a_{1}+b_{1}\right)=\left(c_{2}+d_{2}\right)+\left(a_{1}+b_{1}\right) \\
& \left(\lambda+{ }_{k} \mu\right)(a) \wedge\left(\lambda+_{k} \mu\right)(b) \\
& =\left[\underset{a+\left(a_{1}+b_{1}\right)=\left(a_{2}+b_{2}\right)}{\vee}\left[\lambda\left(a_{1}\right) \wedge \lambda\left(a_{2}\right) \wedge \mu\left(b_{1}\right) \wedge \mu\left(b_{2}\right)\right]\right] \\
& \wedge\left[\underset{b+\left(c_{1}+d_{1}\right)=\left(c_{2}+d_{2}\right)}{\vee}\left[\lambda\left(c_{1}\right) \wedge \lambda\left(c_{2}\right) \wedge \mu\left(d_{1}\right) \wedge \mu\left(d_{2}\right)\right]\right] \\
& =\underset{\substack{a+\left(a_{1}+b_{1}\right)=\left(a_{2}+b_{2}\right) \\
b+\left(c_{1}+d_{1}\right)=\left(c_{2}+d_{2}\right)}}{\vee}\left(\lambda\left(a_{1}\right) \wedge \lambda\left(a_{2}\right) \wedge \mu\left(b_{1}\right) \wedge \mu\left(b_{2}\right) \wedge \lambda\left(c_{1}\right) \wedge \lambda\left(c_{2}\right) \wedge \mu\left(d_{1}\right) \wedge \mu\left(d_{2}\right)\right) \\
& =\underset{\substack{a+\left(a_{1}+b_{1}\right)=\left(a_{2}+b_{2}\right) \\
b+\left(c_{1}+d_{1}\right)=\left(c_{2}+d_{2}\right)}}{\vee}\left(\lambda\left(a_{2}+c_{1}\right) \wedge \lambda\left(a_{1}+c_{2}\right) \wedge \mu\left(b_{2}+d_{1}\right) \wedge \mu\left(b_{1}+d_{2}\right)\right) \\
& \leq \underset{x+\left(a^{\prime}+b^{\prime}\right)=\left(a^{\prime \prime}+b^{\prime \prime}\right)}{\vee}\left[\lambda\left(a^{\prime}\right) \wedge \lambda\left(a^{\prime \prime}\right) \wedge \mu\left(b^{\prime}\right) \wedge \mu\left(b^{\prime \prime}\right)\right]=\left(\lambda+_{k} \mu\right)(x) \text {. }
\end{aligned}
$$


Thus $\lambda+{ }_{k} \mu$ is a fuzzy $k$-ideal of $R$.

\subsection{Theorem}

If $\mu$ is a fuzzy subset of a hemiring $R$, then the following are equivalent:

1) $\mu$ satisfies a) $\mu(x+y) \geq \min \{\mu(x), \mu(y)\}$ and b) $x+a=b \rightarrow \mu(x) \geq \min \{\mu(a), \mu(b)\}$,

2) $\mu+_{k} \mu \leq \mu$.

Proof. 1) $\rightarrow$ 2) Let $x \in R$, then

$$
\begin{aligned}
& \left(\mu+{ }_{k} \mu\right)(x) \\
& =\underset{x+\left(a_{1}+b_{1}\right)=\left(a_{2}+b_{2}\right)}{\vee}\left[\mu\left(a_{1}\right) \wedge \mu\left(a_{2}\right) \wedge \mu\left(b_{1}\right) \wedge \mu\left(b_{2}\right)\right] \\
& \leq \underset{x+\left(a_{1}+b_{1}\right)=\left(a_{2}+b_{2}\right)}{\vee}\left[\mu\left(a_{1}+a_{2}\right) \wedge \mu\left(b_{1}+b_{2}\right)\right] \quad \text { by (1) } \\
& \leq \mu(x)
\end{aligned}
$$

Thus $\mu+{ }_{k} \mu \leq \mu$.

$2) \rightarrow 1)$ First we show that $\mu(0) \geq \mu(x)$ for all $x \in R$.

$$
\begin{aligned}
& \mu(0) \geq\left(\mu++_{k} \mu\right)(0) \\
= & \vee \\
0+\left(a_{1}+b_{1}\right)=\left(a_{2}+b_{2}\right) & {\left[\mu\left(a_{1}\right) \wedge \mu\left(a_{2}\right) \wedge \mu\left(b_{1}\right) \wedge \mu\left(b_{2}\right)\right] } \\
\geq & \mu(x) \wedge \mu(x) \wedge \mu(x) \wedge \mu(x) \\
& \text { because } 0+x+x=x+x \\
= & \mu(x) .
\end{aligned}
$$

Thus $\mu(0) \geq \mu(x)$ for all $x \in R$.

Now

$$
\begin{aligned}
& \mu(x+y) \geq\left(\mu+{ }_{k} \mu\right)(x+y) \\
= & \underset{x+y+\left(a_{1}+b_{1}\right)=\left(a_{2}+b_{2}\right)}{\vee}\left[\mu\left(a_{1}\right) \wedge \mu\left(a_{2}\right) \wedge \mu\left(b_{1}\right) \wedge \mu\left(b_{2}\right)\right] \\
\geq & \mu(0) \wedge \mu(0) \wedge \mu(x) \wedge \mu(y) \\
& \text { because } x+y+0+0=x+y \\
= & \mu(x) \wedge \mu(y) \\
& (\text { because } \mu(0) \geq \mu(x) \text { for all } x \in R) .
\end{aligned}
$$

Again

$$
\begin{aligned}
\mu(x) & \geq\left(\mu+{ }_{k} \mu\right)(x) \\
& =\underset{x+\left(a_{1}+b_{1}\right)=\left(a_{2}+b_{2}\right)}{\vee}\left[\mu\left(a_{1}\right) \wedge \mu\left(a_{2}\right) \wedge \mu\left(b_{1}\right) \wedge \mu\left(b_{2}\right)\right]
\end{aligned}
$$

If $x+a=b$ then $x+a+0=b+0$ and so $\mu(x) \geq \mu(a) \wedge \mu(0) \wedge \mu(b) \wedge \mu(0)=\mu(a) \wedge \mu(b)$ (because $\mu(0) \geq \mu(x)$ for all $x \in R$ ).

\subsection{Lemma}

A fuzzy subset $\mu$ in a hemiring $R$ is a fuzzy left (right) $k$-ideal if and only if

1) $\mu+{ }_{k} \mu \leq \mu$,
2) $\chi_{R} \odot_{k} \mu \leq \mu \quad\left(\mu \odot_{k} \chi_{R} \leq \mu\right)$.

Proof. Let $\mu$ be a fuzzy left $k$-ideal of $R$. By Theorem 3.7, $\mu$ satisfies 1). Now we prove condition 2). Let $x \in R$. If $\left(\chi_{R} \odot_{k} \mu\right)(x)=0$, then

$\left(\chi_{R} \odot_{k} \mu\right)(x) \leq(\mu)(x)$. Otherwise, there exist elements $a_{i}, b_{i}, a_{j}^{\prime}, b_{j}^{\prime} \in R$ such that $x+\sum_{i=1}^{m} a_{i} b_{i}=\sum_{j=1}^{n} a_{j}^{\prime} b_{j}^{\prime}$. Then we have

$$
\begin{aligned}
& \left(\chi_{R} \odot_{k} \mu\right)(x) \\
& =\underset{x+\sum_{i=1}^{m} a_{i} b_{i}=\sum_{j=1}^{n} a_{j}^{\prime} b_{j}^{\prime}}{\vee}\left[\left[\bigwedge_{i=1}^{m} \chi_{R}\left(a_{i}\right)\right] \wedge\left[\bigwedge_{i=1}^{m} \mu\left(b_{i}\right)\right]\right. \\
& \left.\wedge\left[\widehat{ }_{j=1}^{n} \chi_{R}\left(a_{j}^{\prime}\right)\right] \wedge\left[{ }_{j=1}^{n} \mu\left(b_{j}^{\prime}\right)\right]\right] \\
& \left.\leq \underset{x+\sum_{i=1}^{m} a_{i} b_{i}=\sum_{j=1}^{n} a_{b^{\prime}} b_{j}^{\prime}}{\vee}\left[\bigwedge_{i=1}^{m} \mu\left(a_{i} b_{i}\right)\right] \wedge\left[\bigwedge_{j=1}^{n} \mu\left(a_{j}^{\prime} b_{j}^{\prime}\right)\right]\right] \\
& \leq \underset{x+\sum_{i=1}^{m} a_{i} b_{i}=\sum_{j=1}^{n} a_{j}^{\prime} b_{j}^{\prime}}{\vee}\left[\mu\left(\sum_{i=1}^{m} a_{i} b_{i}\right) \wedge \mu\left(\sum_{i=1}^{m} a_{j}^{\prime} b_{j}^{\prime}\right)\right] \\
& \leq \underset{x+\sum_{i=1}^{m} a_{i} b_{i}=\sum_{j=1}^{n} a^{\prime} b_{j}^{\prime} b_{j}^{\prime}}{\vee} \mu(x)=\mu(x) .
\end{aligned}
$$

This implies that $\chi_{R} \odot_{k} \mu \leq \mu$.

Conversely, assume that the given conditions hold. In order to show that $\mu$ is a fuzzy left $k$-ideal of $R$ it is sufficient to show that the condition $\mu(x y) \geq \mu(y)$ holds. Let $x, y \in R$. Then we have

$$
\begin{aligned}
& \mu(x y) \geq\left(\chi_{R} \odot_{k} \mu\right)(x y) \\
& =\underset{x+\sum_{i=1}^{m} a_{i} b_{i}=\sum_{j=1}^{n} a_{j}^{\prime} b_{j}^{\prime}}{\vee}\left[\left[\bigwedge_{i=1}^{m} \mu\left(a_{i}\right)\right] \wedge\left[\bigwedge_{i=1}^{m} \mu\left(b_{i}\right)\right]\right. \\
& \left.\wedge\left[{ }_{j=1}^{n} \mu\left(a_{j}^{\prime}\right)\right] \wedge\left[{ }_{j=1}^{n} \mu\left(b_{j}^{\prime}\right)\right]\right]
\end{aligned}
$$

since $x y+0 y=x y$, so $\mu(x y) \geq \mu(y)$ and $\mu$ is a fuzzy left $k$-ideal of $R$.

For $k$-hemiregular hemirings we have stronger result.

\subsection{Theorem}

A hemiring $R$ is $k$-hemiregular if and only if for any fuzzy right $k$-ideal $\mu$ and any fuzzy left $k$-ideal $v$ of $R$ we have $\mu \odot_{k} v=\mu \wedge v$.

Proof. Let $R$ be a $k$-hemiregular hemiring and $\mu, v$ be fuzzy right $k$-ideal and fuzzy left $k$-ideal of $R$, respectively. Then by Lemma 3.8, we have

$\mu \odot_{k} v \leq \mu \odot_{k} \chi_{R} \leq \mu$ and $\mu \odot_{k} v \leq \chi_{R} \odot_{k} v \leq v$. Thus $\mu \odot_{k} v \leq \mu \wedge v$. To show the converse inclusion, let $x \in R$. Since $R$ is $k$-hemiregular, so there exist $a, a^{\prime} \in R$ such that $x+x a x=x a^{\prime} x$. Then we have 


$$
\begin{aligned}
& \left(\mu \odot_{k} v\right)(x)=\underset{x+\sum_{i=1}^{m} a_{i} b_{i}=\sum_{j=1}^{n} a_{j}^{\prime} b_{j}^{\prime}}{\vee}\left(\left[\bigwedge_{i=1}^{m} \mu\left(a_{i}\right)\right] \wedge\left[\bigwedge_{i=1}^{m} \mu\left(b_{i}\right)\right]\right. \\
& \left.\wedge\left[\widehat{\wedge}_{j=1}^{n} v\left(a_{j}^{\prime}\right)\right] \wedge\left[{ }_{j=1}^{n} v\left(b_{j}^{\prime}\right)\right]\right) \\
& \geq \min \left\{\mu(x a), \mu\left(x a^{\prime}\right), v(x)\right\} \\
& \geq \min \{\mu(x), v(x)\}=(\mu \wedge v)(x) .
\end{aligned}
$$

This implies that $\mu \odot_{k} v \geq \mu \wedge v$. Therefore $\mu \odot_{k} v=\mu \wedge \cap v$.

Conversely, let $C, D$ be any right $k$-ideal and any left $k$-ideal of $R$, respectively. Then the characteristic functions $\chi_{C}, \chi_{D}$ of $C, D$ are fuzzy right $k$-ideal and fuzzy left $k$-ideal of $R$, respectively. Now, by the assumption and Lemma 3.3, we have

$$
\chi_{\overline{C D}}=\chi_{C} \odot_{k} \chi_{D}=\chi_{C} \wedge \chi_{D}=\chi_{C \cap D} .
$$

So, $\overline{C D}=C \cap D$. Hence by Lemma $2.5, R$ is $k$ hemiregular hemiring.

\section{Idempotent $\boldsymbol{k}$-Ideals}

From Lemma 2.5 it follows that in a $k$-hemiregular hemiring every $k$-ideal $A$ is $k$-idempotent, that is $\overrightarrow{A A}=A$. On the other hand, in such hemirings we have $\lambda \odot_{k} \lambda=\lambda$ for all fuzzy $k$-ideals $\lambda$. Fuzzy $k$-ideal with this property will be called idempotent.

\subsection{Proposition}

The following statements are equivalent for a hemiring $R$ :

1) Each $k$-ideal of $R$ is idempotent.

2) $A \cap B=\overline{A B}$ for each pair of $k$-ideals $A, B$ of $R$.

3) $x \in \overline{R x R x R}$ for every $x \in R$.

4) $X \subseteq \overline{R X R X R}$ for every non empty subset $X$ of $R$.

5) $A=\overline{R A R A R}$ for every $k$-ideal $A$ of $R$.

If $R$ is commutative, then the above assertions are equivalent to

6) $R$ is $k$-hemiregular.

Proof. 1) $\rightarrow$ 2) Assume that each $k$-ideal of $R$ is idempotent and $A, B$ are k-ideals of $R$. By Lemma 2.3,

$\overline{A B} \subseteq A \cap B$. Since $A \cap B$ is a $k$-ideal of $R$, so by 1 ) $A \cap B=\overline{(A \cap B)(A \cap B)} \subseteq \overline{A B}$. Thus $A \cap B=\overline{A B}$.

2) $\rightarrow$ 1) Obvious.

1) $\rightarrow 3$ ) Let $x \in \underline{R}$. The smallest $k$-ideal containing $x$ has the form $\overline{\langle x\rangle}=\overline{R x+x R+R x R+\mathbb{N}^{0} x}$, where $\mathbb{N}^{0}$ is the set of whole numbers. By hypothesis

$$
\begin{aligned}
\overline{\langle x\rangle} & =\overline{\overline{\langle x\rangle\langle x\rangle}}=\overline{\langle x\rangle\langle x\rangle} . \text { Thus } \\
x & \in \overline{\left(R x+x R+R x R+\mathbb{N}^{0} x\right)\left(R x+x R+R x R+\mathbb{N}^{0} x\right)} \\
& \subseteq \overline{R x R R x R} \subseteq \overline{R x R x R .}
\end{aligned}
$$

3) $\rightarrow$ 4) This is obvious.

4) $\rightarrow 5$ ) Let $A$ be a $k$-ideal of $R$. Then

$\bar{A}=A \subseteq \overline{R A R A R} \subseteq \overline{A A} \subseteq \bar{A}=A$. Hence $A=\overline{R A R A R}$.

5) $\rightarrow 1$ ) This is obvious.

If $R$ is commutative then by Lemma 2.5, 2) $\leftrightarrow 3$ ) .

\subsection{Proposition}

The following statements are equivalent for a hemiring $R$.

1) Each fuzzy $k$-ideal of $R$ is idempotent.

2) $\lambda \odot_{k} \mu=\lambda \wedge \mu$ for all fuzzy $k$-ideals of $R$.

If $R$ is commutative, then the above assertions are equivalent to

3) $R$ is $k$-hemiregular.

Proof. 1) $\rightarrow$ 2) Let $\lambda$ and $\mu$ be fuzzy $k$-ideals of $R$. By Proposition 3.2, $(\lambda \wedge \mu) \odot_{k}(\lambda \wedge \mu) \leq \lambda \odot_{k} \mu$. Since $\lambda \wedge \mu$ is a fuzzy $k$-ideal of $R$, so by hypothesis $\lambda \wedge \mu$ is idempotent. Thus $\lambda \wedge \mu=(\lambda \wedge \mu) \odot_{k}(\lambda \wedge \mu) \leq \lambda \odot_{k} \mu$. By Theorem 3.4, $\lambda \odot_{k} \mu \leq \lambda \wedge \mu$. Thus $\lambda \odot_{k} \mu=\lambda \wedge \mu$.

2) $\rightarrow$ 1) Obvious.

If $R$ is commutative then by Theorem 3.9, 2) $\leftrightarrow 3$ ) . $\square$

\subsection{Theorem}

Let $R$ be a hemiring with identity 1 , then the following assertions are equivalent:

1) Each $k$-ideal of $R$ is idempotent.

2) $A \cap B=\overline{A B}$ for each pair of $k$-ideals $A, B$ of $R$.

3) Each fuzzy $k$-ideal of $R$ is idempotent.

4) $\lambda \odot_{k} \mu=\lambda \wedge \mu$ for all fuzzy $k$-ideals of $R$.

Proof. 1) $\leftrightarrow$ 2) By Proposition 4.1.

3) $\leftrightarrow$ 4) By Proposition 4.2.

1) $\rightarrow 3$ ) Let $x \in R$. The smallest $k$-ideal of $R$ containing $x$ has the form $\overline{R x R}$. By hypothesis, we have

$\overline{R \times R}=\overline{(\overline{R \times R})(\overline{R \times R})}=\overline{R \times R R \times R}$. Thus

$x \in \overline{R \times R}=\overline{R \times R R \times R}$, this implies

$$
x+\sum_{i=1}^{m} r_{i} x s_{i} u_{i} x t_{i}=\sum_{j=1}^{n} r_{j}^{\prime} x s_{j}^{\prime} u_{j}^{\prime} t_{j}^{\prime}
$$

for some $r_{i}, s_{i}, u_{i}, t_{i}, r_{j}^{\prime}, s_{j}^{\prime}, u_{j}^{\prime}, t_{j}^{\prime} \in R$.

As $\lambda(x) \leq \lambda\left(r_{i} x S_{i}\right)$ and $\lambda(x) \leq \lambda\left(u_{i} x t_{i}\right)$ for each $i \in\{1,2, \cdots, m\}$, so

$$
\lambda(x) \leq \bigwedge_{i=1}^{m} \lambda\left(r_{i} x s_{i}\right) \text { and } \lambda(x) \leq \bigwedge_{i=1}^{m}\left(u_{i} x t_{i}\right) .
$$

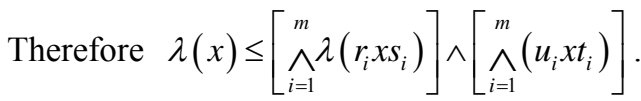

Similarly

$$
\lambda(x) \leq\left[\hat{j}_{j=1}^{n} \lambda\left(r_{j}^{\prime} x s_{j}^{\prime}\right)\right] \wedge\left[\hat{}_{j=1}^{n}\left(u_{j}^{\prime} x t_{j}^{\prime}\right)\right] .
$$

Therefore 


$$
\begin{aligned}
& \lambda(x) \leq\left[\hat{\wedge}_{i=1}^{m} \lambda\left(r_{i} x s_{i}\right)\right] \wedge\left[\hat{\wedge}_{i=1}^{m}\left(u_{i} x t_{i}\right)\right] \wedge\left[\hat{}_{j=1}^{n} \lambda\left(r_{j}^{\prime} x s_{j}^{\prime}\right)\right] \wedge\left[\hat{\jmath}_{j=1}^{n} \lambda\left(u_{j}^{\prime} x t_{j}^{\prime}\right)\right]
\end{aligned}
$$

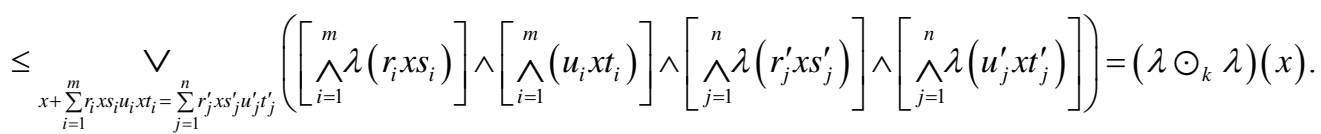

Hence $\lambda \leq \lambda \odot_{k} \lambda$. By Theorem 3.4, $\lambda \odot_{k} \lambda \leq \lambda$. Thus $\lambda \odot_{k} \lambda=\lambda$.

$3) \rightarrow 1$ ) Let $A$ be a $k$-ideal of $R$, then the characteristic function $\chi_{A}$ of $A$ is a fuzzy $k$-ideal of $R$. Hence by hypothesis $\chi_{A}=\chi_{A} \odot_{k} \chi_{A}=\chi_{\overline{A A}}$. Thus $A=\overline{A A}$.

\subsection{Theorem}

If each $k$-ideal of $R$ is idempotent, then the collection of all $k$-ideals of $R$ is a complete Brouwerian lattice.

Proof. Let $\mathcal{L}_{R}$ be the collection of all $k$-ideals of $R$, then $\mathcal{L}_{R}$ is a poset under the inclusion of sets. It is not difficult to see that $\mathcal{L}_{R}$ is a complete lattice under the operations $\sqcup, \sqcap$ defined as $A \sqcup B=\overline{A+B}$ and $A \sqcap B=A \cap B$.

We now show that $\mathcal{L}_{R}$ is a Brouwerian lattice, that is, for any $A, B \in \mathcal{L}_{R}$, the set

$\mathcal{L}_{R}(A, B)=\left\{I \in \mathcal{L}_{R} \mid A \cap I \subseteq B\right\}$ contains a greatest element.

By Zorn's Lemma the set $\mathcal{L}_{R}(A, B)$ contains a maximal element $M$. Since each $k$-ideal of $R$ is idempotent, so $\overline{A I}=A \cap I \subseteq B$ and $\overline{A M}=A \cap M \subseteq B$. Thus $\overline{A I}+\overline{A M} \subseteq B$. Consequently, $\overline{\overline{A I}+\overline{A M}} \subseteq \bar{B}=B$.

Since $\overline{I+M}=I \sqcup M \in \mathcal{L}_{R}$, for every $x \in \overline{I+M}$ there exist $i_{1}, i_{2} \in I, \quad m_{1}, m_{2} \in M \quad$ such that $x+i_{1}+m_{1}=i_{2}+m_{2}$. Thus $d x+d i_{1}+d m_{1}=d i_{2}+d m_{2}$ for any $d \in D \in \mathcal{L}_{R}$. As $d i_{1}, d i_{2} \in D I, \quad d m_{1}, d m_{2} \in D M$, we have $d x \in \overline{D I+D M}$, which implies

$$
D(\overline{I+M}) \subseteq \overline{D I+D M} \subseteq \overline{\overline{D I}+\overline{D M}} \subseteq B .
$$

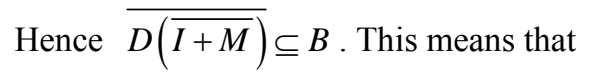

$D \bigcap(\overline{I+M})=\overline{D(\overline{I+M})} \subseteq B$, i.e., $\overline{I+M} \in \mathcal{L}_{R}(A, B)$, whence $\overline{I+M}=M$ because $M$ is maximal in $\mathcal{L}_{R}(A, B)$. Therefore $I \subseteq \bar{I} \subseteq \overline{I+M}=M$ for every $I \in \mathcal{L}_{R}(A, B)$.

\subsection{Corollary}

If each $k$-ideal of $R$ is idempotent, then the lattice $\mathcal{L}_{R}$ of all $k$-ideal of $R$ is distributive.

Proof. Each complete Brouwerian lattice is distributive (cf. [31], 11.11).

\subsection{Theorem}

Each fuzzy $k$-ideal of $R$ is idempotent if and only if the set of all fuzzy $k$-ideal of $R$ (ordered by $\leq$ ) forms a distributive lattice under the $k$-sum and $k$-product of fuzzy $k$-ideals with $\lambda \odot_{k} \mu=\lambda \wedge \mu$.

Proof. Suppose that each fuzzy $k$-ideal of $R$ is idempotent. Then by Proposition 4.2, $\lambda \odot_{k} \mu=\lambda \wedge \mu$. Let $\mathcal{F} \mathcal{L}_{R}$ be the collection of all fuzzy $k$-ideals of $R$. Then $\mathcal{F} \mathcal{L}_{R}$ is a lattice (ordered by $\leq$ ) under the $k$-sum and $k$-product of fuzzy $k$-ideals.

We show that $\left(\lambda \odot_{k} \delta\right)+_{k} \mu=\left(\lambda+_{k} \mu\right) \odot_{k}\left(\delta+_{k} \mu\right)$ for all $\lambda, \mu, \delta \in \mathcal{F} \mathcal{L}_{R}$. Let $x \in R$, then

$$
\begin{aligned}
& \left(\left(\lambda \odot_{k} \delta\right)+{ }_{k} \mu\right)(x)=\underset{x+\left(a_{1}+b_{1}\right)=\left(a_{2}+b_{2}\right)}{\vee}\left[(\lambda \wedge \delta)\left(a_{1}\right) \wedge(\lambda \wedge \delta)\left(a_{2}\right) \wedge \mu\left(b_{1}\right) \wedge \mu\left(b_{2}\right)\right] \\
& =\underset{x+\left(a_{1}+b_{1}\right)=\left(a_{2}+b_{2}\right)}{\vee}\left[\lambda\left(a_{1}\right) \wedge \lambda\left(a_{2}\right) \wedge \mu\left(b_{1}\right) \wedge \mu\left(b_{2}\right) \wedge \delta\left(a_{1}\right) \wedge \delta\left(a_{2}\right)\right] \\
& =\left[\underset{x+\left(a_{1}+b_{1}\right)=\left(a_{2}+b_{2}\right)}{\vee}\left[\lambda\left(a_{1}\right) \wedge \lambda\left(a_{2}\right) \wedge \mu\left(b_{1}\right) \wedge \mu\left(b_{2}\right)\right]\right] \wedge\left[\underset{x+\left(a_{1}+b_{1}\right)=\left(a_{2}+b_{2}\right)}{\vee}\left[\delta\left(a_{1}\right) \wedge \delta\left(a_{2}\right) \wedge \mu\left(b_{1}\right) \wedge \mu\left(b_{2}\right)\right]\right] \\
& =\left(\lambda+{ }_{k} \mu\right)(x) \wedge\left(\delta+{ }_{k} \mu\right)(x)=\left[\left(\lambda+{ }_{k} \mu\right) \wedge\left(\delta+{ }_{k} \mu\right)\right](x)=\left(\left(\lambda+{ }_{k} \mu\right) \odot_{k}\left(\delta+{ }_{k} \mu\right)\right)(x) .
\end{aligned}
$$

So, $\mathcal{F} \mathcal{L}_{R}$ is a distributive lattice.

The converse is obvious.

\section{Prime $\boldsymbol{k}$-Ideals}

A proper (left, right) $k$-ideal $P$ of $R$ is called prime if for any (left, right) $k$-ideals $A, B$ of $R, A B \subseteq P$ implies $A \subseteq P$ or $B \subseteq P$. A proper (left, right) $k$-ideal $P$ of $R$ is called irreducible if for any (left, right) $k$-ideals $A, B$ of $R$, $A \cap B=P$ implies $A=P$ or $B=P$. By analogy a non-constant fuzzy $k$-ideal $\delta$ of $R$ is called prime (in the first sense) if for any fuzzy $k$-ideals $\lambda, \mu$ of $R$, $\lambda \odot_{k} \mu \leq \delta$ implies $\lambda \leq \delta$ or $\mu \leq \delta$, and irreducible if $\lambda \wedge \mu=\delta$ implies $\lambda=\delta$ or $\mu=\delta$.

\subsection{Theorem}

A left (right) $k$-ideal $P$ of a hemiring $R$ with identity is prime if and only if for all $a, b \in R$ from $a R b \subseteq P$ it follows $a \in P$ or $b \in P$. 
Proof. Assume that $P$ is a prime left $k$-ideal of $R$ and $a R b \subseteq P$ for some $a, b \in R$. Obviously, $A=\overline{R a}$ and $B=\overline{R b}$ are left $k$-ideals of $R$ generated by $a$ and $b$, respectively. So, $A B \subseteq \overline{A B}=\overline{\overline{R a} \overline{R b}}=\overline{R a R b} \subseteq \overline{R P} \subseteq P$, and consequently $A \subseteq P$ or $B \subseteq P$. If $A \subseteq P$, then $a \in P$. If $B \subseteq P$, then $b \in P$.

The converse is obvious.

\subsection{Corollary}

A $k$-ideal $P$ of a hemiring $R$ with identity is prime if and only if for all $a, b \in R$ from $a R b \subseteq P$ it follows $a \in P$ or $b \in P$.

\subsection{Corollary}

A $k$-ideal $P$ of a commutative hemiring $R$ with identity is prime if and only if for all $a, b \in R$ from $a b \in P$ it follows $a \in P$ or $b \in P$.

The result expressed by Corollary 5.3, suggests the following definition of prime fuzzy $k$-ideals.

\subsection{Definition}

A non-constant fuzzy $k$-ideal $\delta$ of $R$ is called prime (in the second sense) if for all $t \in[0,1]$ and $a, b \in R$ the following condition is satisfied:

$$
\begin{gathered}
\text { if } \delta(a x b) \geq t \text { for every } x \in R \text { then } \\
\delta(a) \geq t \text { or } \delta(b) \geq t .
\end{gathered}
$$

In other words, a non-constant fuzzy $k$-ideal $\delta$ is prime if from the fact that $a x b \in U(\delta ; t)$ for every $x \in R$ it follows $a \in U(\delta ; t)$ or $b \in U(\delta ; t)$. It is clear that any fuzzy $k$-ideal is prime in the first sense is prime in the second sense. The converse is not true.

\subsection{Example}

In an ordinary hemiring of natural numbers the set of even numbers forms a $k$-ideal. A fuzzy set

$$
\delta(n)= \begin{cases}1 & \text { if } n=0, \\ 0.5 & \text { if } n=2 k \neq 0, \\ 0.3 & \text { if } n=2 k+1\end{cases}
$$

is a fuzzy $k$-ideal of this hemiring. It is prime in the second sense but it is not prime in the first sense.

\subsection{Theorem}

A non-constant fuzzy $k$-ideal $\delta$ of a hemiring $R$ with identity is prime in the second sense if and only if each its proper level set $U(\delta ; t)$ is a prime $k$-ideal of $R$.

Proof. Suppose $\delta$ is a prime fuzzy $k$-ideal of $R$ in the second sense and let $U(\delta ; t)$ be its arbitrary proper level set, i.e., $\varnothing \neq U(\delta ; t) \neq R$. If $a R b \subseteq U(\delta ; t)$, then $\delta(a x b) \geq t$ for every $x \in R$. Hence $\delta(a) \geq t$ or $\delta(b) \geq t$, i.e., $a \in U(\delta ; t)$ or $b \in U(\delta ; t)$, which, by Corollary 5.3 , means that $U(\delta ; t)$ is a prime $k$-ideal of R.

To prove the converse, consider a non-constant fuzzy $k$-ideal $\delta$ of $R$. If it is not prime then there exist $a$, $b \in R$ such that $\delta(a x b) \geq t$ for all $x \in R$, but $\delta(a)<t$ and $\delta(b)<t$. Thus, $a R b \subseteq U(\delta ; t)$, but $a \notin U(\delta ; t)$ and $b \notin U(\delta ; t)$. Therefore $U(\delta ; t)$ is not prime, which is a contradiction. Hence $\delta$ is a prime fuzzy $k$-ideal in the second sense.

\subsection{Corollary}

The fuzzy set $\lambda_{A}$ defined in Proposition 2.8 , is a prime fuzzy $k$-ideal of $R$ (with identity) in the second sense if and only if $A$ is a prime $k$-ideal of $R$.

In view of the Transfer Principle the second definition of prime fuzzy $k$-ideal is better. Therefore fuzzy $k$-ideals which are prime in the first sense will be called $k$-prime.

\subsection{Proposition}

A non-constant fuzzy $k$-ideal $\delta$ of a commutative hemiring $R$ with identity is prime if and only if $\delta(a b)=\delta(a) \vee \delta(b)$ for all $a, b \in R$.

Proof. Let $\delta$ be a non-constant fuzzy $k$-ideal of a commutative hemiring $R$ with identity. If $\delta(a b)=t$, then for every $x \in R$, we have $\delta(a x b)=\delta(x a b) \geq \delta(x) \vee \delta(a b) \geq t$. Thus $\delta(a x b) \geq t$ for every $x \in R$, which implies $\delta(a) \geq t$ or $\delta(b) \geq t$. If $\delta(a) \geq t$, then $t=\delta(a b) \geq \delta(a) \geq t$, whence $\delta(a b)=\delta(a)$. If $\delta(b) \geq t$, then, as in the previous case, $\delta(a b)=\delta(b)$. So, $\delta(a b)=\delta(a) \vee \delta(b)$.

Conversely, assume that $\delta(a b)=\delta(a) \vee \delta(b)$ for all $a, b \in R$. If $\delta(a x b) \geq t$ for every $x \in R$, then replacing $x$ by the identity of $R$, we obtain $\delta(a b) \geq t$. Thus $\delta(a) \vee \delta(b) \geq t$, i.e., $\delta(a) \geq t$ or $\delta(b) \geq t$, which means that $\delta$ is prime.

\subsection{Theorem}

Every proper $k$-ideal of a hemiring $R$ is contained in some proper irreducible $k$-ideal of $R$.

Proof. Let $P$ be a proper $k$-ideal of $R$ such that $a \notin P$. Let $\left\{P_{\alpha} \mid \alpha \in \Lambda\right\}$ be a family of all proper $k$-ideals of $R$ containing $P$ and not containing $a$. By Zorn's Lemma, this family contains a maximal element, say $M$. This maximal element is an irreducible $k$-ideal. Indeed, let $M=P_{\beta} \cap P_{\delta}$ for some $k$-ideals $P_{\beta}, P_{\delta}$ of $R$. If $M$ is a proper subset of $P_{\beta}$ and $P_{\delta}$, then, according to the maximality of $M$, we have $a \in P_{\beta}$ and $a \in P_{\delta}$. Hence $a \in P_{\beta} \cap P_{\delta}=M$, which is impossible. Thus, either $M=P_{\beta}$ or $M=P_{\delta}$. 


\subsection{Theorem}

If all $k$-ideals of $R$ are idempotent, then a $k$-ideal $P$ of $R$ is irreducible if and only if it is prime.

Proof. Assume that all $k$-ideals of $R$ are idempotent. Let $P$ be a fixed irreducible $k$-ideal. If $A B \subseteq P$ for some $k$-ideals $A, B$ of $R$, then by Proposition 4.1, $A \cap B=\overline{A B} \subseteq \bar{P}=P$. Thus $\overline{(A \cap B)+P}=P$. Since $\mathcal{L}_{R}$ is a distributive lattice, so

$$
P=\overline{(A \cap B)+P}=\overline{(A+P)} \cap \overline{(B+P)} .
$$

So either $\overline{A+P}=P$ or $\overline{B+P}=P$, that is either $A \subseteq P$ or $B \subseteq P$.

Conversely, if a $k$-ideal $P$ is prime and $A \cap B=P$ for some $A, B \in \mathcal{L}_{R}$, then $A B \subseteq \overline{A B}=A \cap B=P$. Thus $A \subseteq P$ or $B \subseteq P$. But $P \subseteq A$ and $P \subseteq B$. Hence $A=P$ or $B=P$.

\subsection{Corollary}

Let $R$ be a hemiring in which all $k$-ideals are idempotent. Then each proper $k$-ideal of $R$ is contained in some proper prime $k$-ideal.

\subsection{Theorem}

Let $R$ be a hemiring in which all fuzzy $k$-ideals are idempotent. Then a fuzzy $k$-ideal of $R$ is irreducible if and only if it is $k$-prime.

Proof. Assume that all fuzzy $k$-ideals of $R$ are idempotent and let $\delta$ be an arbitrary irreducible fuzzy $k$-ideal of $R$. We prove that it is $k$-prime. If $\lambda \odot_{k} \mu \leq \delta$ for some fuzzy $k$-ideals $\lambda, \mu$ of $R$ then also $\lambda \wedge \mu \leq \delta$. Since the set $\mathcal{F} \mathcal{L}_{R}$ of all fuzzy $k$-ideals of $R$ is a distributive lattice, we have

$\delta=(\lambda \wedge \mu)+_{k} \delta=\left(\lambda+_{k} \delta\right) \wedge\left(\mu+_{k} \delta\right)$. Thus $\lambda+_{k} \delta=\delta$ or $\mu+{ }_{k} \delta=\delta$. Thus $\lambda \leq \delta$ or $\mu \leq \delta$. This proves that $\delta$ is $k$-prime.

Conversely, if $\delta$ is a $k$-prime fuzzy $k$-ideal of $R$ and $\lambda \wedge \mu=\delta$ for some $\lambda, \mu \in \mathcal{F} \mathcal{L}_{R}$, then $\lambda \odot_{k} \mu=\delta$, which implies $\lambda \leq \delta$ or $\mu \leq \delta$. Since $\delta=\lambda \wedge \mu$, so we have also $\delta \leq \lambda$ and $\delta \leq \mu$. Thus $\lambda=\delta$ or $\mu=\delta$. So, $\delta$ is irreducible.

\subsection{Theorem}

The following assertions for a hemiring $R$ are equivalent:

1) Each $k$-ideal of $R$ is idempotent.

2) Each proper $k$-ideal $P$ of $R$ is the intersection of all prime $k$-ideals of $R$ which contain $P$.

Proof. 1) $\rightarrow$ 2) Let $P$ be a proper $k$-ideal of $R$ and let $\left\{P_{\alpha} \mid \alpha \in \Lambda\right\}$ be the family of all prime $k$-ideals of $R$ which contain $P$. Theorem 5.9, guarantees the existance of such ideals. Clearly $P \subseteq \bigcap_{\alpha \in \Lambda} P_{\alpha}$. If $a \notin P$ then by Theorem 5.9, there exists an irreducible $k$-ideal $P_{a}$ such that $P \subseteq P_{a}$ and $a \notin P_{a}$. By Theorem 5.10, $P_{a}$ is prime. So there exists a prime $k$-ideal $P_{a}$ such that $a \notin P_{a}$ and $P \subseteq P_{a}$. Hence $\cap P_{\alpha} \subseteq P$. Thus $P=\cap P_{\alpha}$.

$2) \rightarrow 1$ ) Assume that each $k$-ideal of $R$ is the intersection of all prime $k$-ideals of $R$ which contain it. Let $A$ be a $k$-ideal of $R$. If $\overline{A^{2}}=R$, then we have $A=R$, which means that $A$ is idempotent. If $\overline{A^{2}} \neq R$, then $\overline{A^{2}}$ is a proper $k$-ideal of $R$ and so it is the intersection of all prime $k$-ideals of $R$ containing $\overline{A^{2}}$. Let $\overline{A^{2}}=\cap P_{\alpha}$. Then $A^{2} \subseteq P_{\alpha}$ for each $\alpha$. Since $P_{\alpha}$ is prime, we have $A \subseteq P_{\alpha}$. Thus $A \subseteq \cap P_{\alpha}=\overline{A^{2}}$. But $\overline{A^{2}} \subseteq A$. Hence $A=\overline{A^{2}}$.

\subsection{Lemma}

Let $R$ be a hemiring in which each fuzzy $k$-ideal is idempotent. If $\lambda$ is a fuzzy $k$-ideal of $R$ with $\lambda(a)=\alpha$, where $a$ is any element of $R$ and $\alpha \in[0,1]$, then there exists an irreducible $k$-prime fuzzy $k$-ideal $\delta$ of $R$ such that $\lambda \leq \delta$ and $\delta(a)=\alpha$.

Proof. Let $\lambda$ be an arbitrary fuzzy $k$-ideal of $R$ and $a \in R$ be fixed. Consider the following collection of fuzzy $k$-ideals of $R$

$$
\mathcal{B}=\{\mu \mid \mu(a)=\lambda(a), \lambda \leq \mu\} .
$$

$\mathcal{B}$ is non-empty since $\lambda \in \mathcal{B}$. Let $\mathcal{F}$ be a totally ordered subset of $\mathcal{B}$ containing $\lambda$, say $\mathcal{F}=\left\{\lambda_{i} \mid i \in I\right\}$.

We claim that $\underset{i \in I}{\vee} \lambda_{i}$ is a fuzzy $k$-ideal of $R$.

For any $x, y \in R$, we have

$$
\begin{aligned}
& \left(\underset{i \in I}{\vee} \lambda_{i}\right)(x) \wedge\left(\underset{i \in I}{\vee} \lambda_{i}\right)(y) \\
& =\left(\underset{i \in I}{\vee} \lambda_{i}(x)\right) \wedge\left(\bigvee_{j \in I} \lambda_{j}(y)\right)=\underset{i, j \in I}{\vee}\left(\lambda_{i}(x) \wedge \lambda_{j}(y)\right) \\
& \leq \underset{i, j \in I}{\vee}\left(\left(\lambda_{i}(x) \vee \lambda_{j}(x)\right) \wedge\left(\lambda_{i}(y) \vee \lambda_{j}(y)\right)\right) \\
& \leq \underset{i, j \in I}{\vee}\left(\lambda_{i}(x+y) \vee \lambda_{j}(x+y)\right) \\
& \leq \underset{i \in I}{\vee} \lambda_{i}(x+y)=\left(\underset{i \in I}{\vee} \lambda_{i}\right)(x+y) .
\end{aligned}
$$

Similarly

$$
\left(\bigvee_{i \in I} \lambda_{i}\right)(x)=\bigvee_{i \in I} \lambda_{i}(x) \leq \bigvee_{i \in I} \lambda_{i}(x r)=\left(\bigvee_{i \in I} \lambda_{i}\right)(x r)
$$

and

$$
\left(\bigvee_{i \in I} \lambda_{i}\right)(x) \leq\left(\bigvee_{i \in I} \lambda_{i}\right)(r x)
$$

for all $x, r \in R$. Thus $\underset{i \in I}{\vee} \lambda_{i}$ is a fuzzy ideal.

Now, let $x+a=b$, where $a, b \in R$. Then 


$$
\begin{aligned}
& \left(\underset{i \in I}{\vee} \lambda_{i}\right)(a) \wedge\left(\bigvee_{i \in I} \lambda_{i}\right)(b) \\
& =\left(\bigvee_{i \in I} \lambda_{i}(a)\right) \wedge\left(\bigvee_{j \in I}\left(\lambda_{j}(b)\right)=\bigvee_{i, j \in I}\left(\lambda_{i}(a) \wedge \lambda_{j}(b)\right)\right. \\
& \leq \underset{i, j \in I}{\vee}\left(\lambda_{i}(a) \vee \lambda_{j}(a)\right) \wedge\left(\lambda_{i}(b) \vee \lambda_{j}(b)\right) \\
& \leq \underset{i, j}{\vee}\left(\lambda_{i}(x) \vee \lambda_{j}(x)\right) \leq \bigvee_{i \in i} \lambda_{i}(x)=\left(\bigvee_{i \in I} \lambda_{i}\right)(x) .
\end{aligned}
$$

Thus $\underset{i \in I}{\vee} \lambda_{i}$ is a fuzzy $k$-ideal of $R$. Clearly $\lambda \leq \underset{i \in I}{\vee} \lambda_{i}$ and $\left(\bigvee_{i \in I} \lambda_{i}\right)(a)=\lambda(a)=\alpha$. Thus $\underset{i \in I}{\vee} \lambda_{i}$ is the least upper bound of $\mathcal{F}$. Hence by Zorn's lemma there exists a fuzzy $k$-ideal $\delta$ of $R$ which is maximal with respect to the property that $\lambda \leq \delta$ and $\delta(a)=\alpha$.

We will show that $\delta$ is an irreducible fuzzy $k$-ideal of $R$. Let $\delta=\delta_{1} \wedge \delta_{2}$, where $\delta_{1}, \delta_{2}$ are fuzzy $k$-ideals of $R$. Then $\delta \leq \delta_{1}$ and $\delta \leq \delta_{2}$. We claim that either $\delta=\delta_{1}$ or $\delta=\delta_{2}$. Suppose $\delta \neq \delta_{1}$ and $\delta \neq \delta_{2}$. Since $\delta$ is maximal with respect to the property that $\delta(a)=\alpha$ and since $\delta \supsetneqq \delta_{1}$ and $\delta \supsetneqq \delta_{2}$, so $\delta_{1}(a) \neq \alpha$ and $\delta_{2}(a) \neq \alpha$. Hence

$$
\alpha=\delta(a)=\left(\delta_{1} \wedge \delta_{2}\right)(a)=\delta_{1}(a) \wedge \delta_{2}(a) \neq \alpha,
$$

which is impossible. Hence $\delta=\delta_{1}$ or $\delta=\delta_{2}$. Thus $\delta$ is an irreducible fuzzy $k$-ideal of $R$. By Theorem 5.12, $\delta$ is $k$-prime.

\subsection{Theorem}

Each fuzzy $k$-ideal of $R$ is idempotent if and only if each fuzzy $k$-ideal of $R$ is the intersection of those $k$-prime fuzzy $k$-ideals of $R$ which contain it.

Proof. Suppose each fuzzy $k$-ideal of $R$ is idempotent. Let $\lambda$ be a fuzzy $k$-ideal of $R$ and let $\left\{\lambda_{\alpha} \mid \alpha \in \Lambda\right\}$ be the family of all $k$-prime fuzzy $k$-ideals of $R$ which contain $\lambda$. Obviously $\lambda \leq \bigwedge_{\alpha \in \Lambda} \lambda_{\alpha}$. We now show that $\widehat{\wedge}_{\alpha \in \Lambda} \lambda_{\alpha} \leq \lambda$. Let $a$ be an arbitrary element of $R$. Then, by Lemma 5.14 , there exists an irreducible $k$-prime fuzzy $k$-ideal $\delta$ such that $\lambda \leq \delta$ and $\lambda(a)=\delta(a)$. Hence $\delta \in\left\{\lambda_{\alpha} \mid \alpha \in \Lambda\right\}$ and $\bigwedge_{\alpha \in \Lambda} \lambda_{\alpha} \leq \delta$. So,

$\bigwedge_{\alpha \in \Lambda} \lambda_{\alpha}(a) \leq \delta(a)=\lambda(a)$. Thus $\bigwedge_{\alpha \in \Lambda} \lambda_{\alpha} \leq \lambda$. Therefore $\bigwedge_{\alpha \in \Lambda} \lambda_{\alpha}=\lambda$.

Conversely, assume that each fuzzy $k$-ideal of $R$ is the intersection of those $k$-prime fuzzy $k$-ideals of $R$ which contain it. Let $\lambda$ be a fuzzy $k$-ideal of $R$ then $\lambda \odot_{k} \lambda$ is also a fuzzy $k$-ideal of $R$, so $\lambda \odot_{k} \lambda=\bigwedge_{\alpha \in \Lambda} \lambda_{\alpha}$ where $\lambda_{\alpha}$ are $k$-prime fuzzy $k$-ideals of $R$. Thus each $\lambda_{\alpha}$ con- tains $\lambda \odot_{k} \lambda$, and hence $\lambda$. So $\lambda \leq \bigwedge_{\alpha \in \Lambda} \lambda_{\alpha}=\lambda \odot \lambda$, but $\lambda \odot_{k} \lambda \leq \lambda$ always. Hence $\lambda=\lambda \odot_{k} \lambda$.

\section{Semiprime $k$-Ideals}

\subsection{Definition}

A proper (left, right) $k$-ideal $A$ of $R$ is called semiprime if for any (left, right) $k$-ideal $B$ of $R, B^{2} \subseteq A$ implies $B \subseteq A$. Similarly, a non-constant fuzzy $k$-ideal $\lambda$ of $R$ is called semiprime if for any fuzzy $k$-ideal $\delta$ of $R$, $\delta \odot_{k} \delta \leq \lambda$ implies $\delta \leq \lambda$.

\subsection{Theorem}

A (left, right) $k$-ideal $P$ of a hemiring $R$ with identity is semiprime if and only if for every $a \in R$ from $a R a \subseteq P$ it follows $a \in P$.

Proof. Proof is similar to the proof of Theorem 5.1.

\subsection{Corollary}

A $k$-ideal $P$ of a commutative hemiring $R$ with identity is semiprime if and only if for all $a \in R$ from $a^{2} \in P$ it follows $a \in P$.

\subsection{Theorem}

The following assertions for a hemiring $R$ are equivalent:

1) Each $k$-ideal of $R$ is idempotent.

2) Each $k$-ideal of $R$ is semiprime.

Proof. Suppose that each $k$-ideal of $R$ is idempotent. Let $A, B$ be $k$-ideals of $R$ such that $B^{2} \subseteq A$. Then $\overline{B^{2}} \subseteq \bar{A}=A$. By hypothesis $B=\overline{B^{2}}$, so $B \subseteq A$. Hence $A$ is semiprime.

Conversely, assume that each $k$-ideal of $R$ is semiprime. Let $A$ be a $k$-ideal of $R$, then $\overline{A^{2}}$ is a $k$-ideal of $R$. Also $A^{2} \subseteq \overline{A^{2}}$. Hence by hypothesis $A \subseteq \overline{A^{2}}$. But $\overline{A^{2}} \subseteq A$ always. Hence $A=\overline{A^{2}}$.

\subsection{Theorem}

Each fuzzy $k$-ideal of $R$ is idempotent if and only if each fuzzy $k$-ideal of $R$ is semiprime.

Proof. For any fuzzy $k$-ideal $\lambda$ of $R$ we have $\lambda \odot_{h} \lambda \leq \lambda$. If each fuzzy $k$-ideal of $R$ is semiprime, then $\lambda \odot_{k} \lambda \leq \lambda \odot_{k} \lambda$ implies $\lambda \leq \lambda \odot_{k} \lambda$. Hence $\lambda \odot_{k} \lambda=\lambda$.

The converse is obvious.

Theorem 6.2, suggest the following definition of semiprime fuzzy $k$-ideals.

\subsection{Definition}

A non-constant fuzzy $k$-ideal $\delta$ of $R$ is called semi- 
prime (in the second sense) if for all $t \in[0,1]$ and $a \in R$ the following condition is satisfied:

if $\delta(a x a) \geq t$ for every $x \in R$ then $\delta(a) \geq t$.

\subsection{Theorem}

A non-constant fuzzy $k$-ideal $\delta$ of $R$ is semiprime in the second sense if and only if each its proper level set $U(\delta ; t)$ is a semiprime $k$-ideal of $R$.

Proof. Proof is similar to the proof of Theorem 5.6.

\subsection{Corollary}

A fuzzy set $\lambda_{A}$ defined in Proposition 2.8 is a semiprime fuzzy $k$-ideal of $R$ in the second sense if and only if $A$ is a semiprime $k$-ideal of $R$.

In view of the Transfer Principle the second definition of semiprime fuzzy $k$-ideal is better. Therefore fuzzy $k$ ideals which are semiprime in the first sense should be called $k$-semiprime.

\subsection{Proposition}

A non-constant fuzzy $k$-ideal $\delta$ of a commutative hemiring $R$ with identity is semiprime if and only if $\delta\left(a^{2}\right)=\delta(a)$ for every $a \in R$.

Proof. Proof is similar to the proof of Proposition 5.8.

Every fuzzy $k$-prime $k$-ideal is fuzzy $k$-semiprime $k$ ideal but the converse is not true.

\subsection{Example}

Consider the hemiring $R=\{0, a, b, c\}$ defined by the following tables:

\begin{tabular}{ccccc}
\hline+ & 0 & $a$ & $b$ & $c$ \\
\hline 0 & 0 & $a$ & $b$ & $c$ \\
$a$ & $a$ & $b$ & $c$ & $a$ \\
$b$ & $b$ & $c$ & $a$ & $b$ \\
$c$ & $c$ & $a$ & $b$ & $c$ \\
\hline$\cdot$ & & & & \\
\hline 0 & 0 & $a$ & $b$ & $c$ \\
\hline$a$ & 0 & 0 & 0 & 0 \\
$b$ & 0 & $a$ & $b$ & $c$ \\
$c$ & 0 & $b$ & $b$ & $c$ \\
\hline
\end{tabular}

This hemiring has two $k$-ideals $\{0, c\}$ and $R$. Obviously these $k$-ideals are idempotent.

For any fuzzy ideal $\lambda$ of $R$ and any $x \in R$ we have $\lambda(0) \geq \lambda(x) \geq \lambda(a)$. Indeed,

$$
\lambda(0)=\lambda(0 x) \geq \lambda(x)=\lambda(x a) \geq \lambda(a) .
$$

This together with

$$
\lambda(a)=\lambda(b+b) \geq \lambda(b) \wedge \lambda(b)=\lambda(b)
$$

implies $\lambda(a)=\lambda(b)$. Consequently,

$$
\lambda(c)=\lambda(a+b) \geq \lambda(a) \wedge \lambda(b)=\lambda(b) .
$$

Therefore $\lambda(0) \geq \lambda(c) \geq \lambda(b)=\lambda(a)$ for every fuzzy $k$-ideal of this hemiring.

Now we prove that each fuzzy $k$-ideal of $R$ is idempotent. Since $\lambda \odot_{k} \lambda \leq \lambda$ always, so we have to show that $\lambda \odot_{k} \lambda \geq \lambda$. Obviously, for every $x \in R$ we have

$$
\begin{aligned}
& \left(\lambda \odot_{k} \lambda\right)(x) \\
& =\sup _{x+\sum_{i=1}^{m} a_{i} b_{i}=\sum_{j=1}^{n} a_{j}^{\prime} b_{j}^{\prime}}\left[\hat{}_{i=1}^{n}\left(\lambda\left(a_{i}\right) \wedge \lambda\left(b_{i}\right)\right) \wedge \hat{\wedge}_{j=1}^{m}\left(\lambda\left(a_{j}^{\prime}\right) \wedge \lambda\left(b_{j}^{\prime}\right)\right)\right] \\
& \geq \sup _{x+c d=c^{\prime} d^{\prime}}\left[\lambda(c) \wedge \lambda(d) \wedge \lambda\left(c^{\prime}\right) \wedge \lambda\left(d^{\prime}\right)\right] \\
& =\lambda(c) \wedge \lambda(d) \wedge \lambda\left(c^{\prime}\right) \wedge \lambda\left(d^{\prime}\right) .
\end{aligned}
$$

So, $x+c d=c^{\prime} d^{\prime}$ implies

$$
\left(\lambda \odot_{k} \lambda\right)(x) \geq \lambda(c) \wedge \lambda(d) \wedge \lambda\left(c^{\prime}\right) \wedge \lambda\left(d^{\prime}\right) .
$$

Hence $0+00=00$ implies $\left(\lambda \odot_{k} \lambda\right)(0) \geq \lambda(0)$. Similarly $a+b b=b c$ implies

$$
\left(\lambda \odot_{k} \lambda\right)(a) \geq \lambda(b) \wedge \lambda(c)=\lambda(b)=\lambda(a),
$$

$b+a a=b c$ implies

$$
\left(\lambda \odot_{k} \lambda\right)(b) \geq \lambda(a) \wedge \lambda(b) \wedge \lambda(c)=\lambda(b) .
$$

Analogously, from $c+00=c c$ it follows

$$
\left(\lambda \odot_{k} \lambda\right)(c) \geq \lambda(0) \wedge \lambda(c)=\lambda(c) .
$$

This proves that $\left(\lambda \odot_{k} \lambda\right)(x) \geq \lambda(x)$ for every $x \in R$. Therefore $\lambda \odot_{k} \lambda=\lambda$ for every fuzzy $k$-ideal of $R$, which, by Theorem 6.4 , means that each fuzzy $k$-ideal of $R$ is semiprime.

Consider the following three fuzzy sets:

$$
\begin{array}{ll}
\lambda(0)=\lambda(c)=0.8, & \lambda(a)=\lambda(b)=0.4, \\
\mu(0)=\mu(c)=0.6, & \mu(a)=\mu(b)=0.5, \\
\delta(0)=\delta(c)=0.7, & \delta(a)=\delta(b)=0.45 .
\end{array}
$$

These three fuzzy sets are idempotent fuzzy $k$-ideals. Since all fuzzy $k$-ideal of this hemiring are idempotent, by Proposition 4.1, we have $\lambda \odot_{k} \mu=\lambda \wedge \mu$. Thus

$$
\left(\lambda \odot_{k} \mu\right)(0)=\left(\lambda \odot_{k} \mu\right)(c)=0.6
$$

and

$$
\left(\lambda \odot_{k} \mu\right)(a)=\left(\lambda \odot_{k} \mu\right)(b)=0.4 .
$$

So, $\lambda \odot_{k} \mu \leq \delta$ but neither $\lambda \leq \delta$ nor $\mu \leq \delta$, that is $\delta$ is not a $k$-prime fuzzy $k$-ideal. 


\section{Prime Spectrum}

Let $R$ be a hemiring in which each $k$-ideal is idempotent. Let $\mathcal{L}(R)$ be the lattice of all $k$-ideals of $R$ and $\mathcal{P}(R)$ be the set of all proper prime $k$-ideals of $R$. For each $k$-ideal $I$ of $R$ define $\theta_{I}=\{J \in \mathcal{P}(R): I \nsubseteq J\}$ and $\mathfrak{I}(\mathcal{P}(R))=\left\{\theta_{I}: I \in \mathcal{L}(R)\right\}$.

\subsection{Theorem}

The set $\mathfrak{I}(\mathcal{P}(R))$ forms a topology on the set $\mathcal{P}(R)$.

Proof. Since $\theta_{\{0\}}=\{J \in \mathcal{P}(R):\{0\} \nsubseteq J\}=\phi$, where $\phi$ is the usual empty set, because 0 belongs to each $k$-ideal. So empty set belongs to $\mathfrak{I}(\mathcal{P}(R))$.

Also $\theta_{R}=\{J \in \mathcal{P}(R): R \nsubseteq J\}=\mathcal{P}(R)$, because $\mathcal{P}(R)$ is the set of all proper prime $k$-ideals of $R$. Thus $\mathcal{P}(R)$ belongs to $\mathfrak{I}(\mathcal{P}(R))$.

Suppose $\theta_{I_{1}}, \theta_{I_{2}} \in \mathfrak{I}(\mathcal{P}(R))$ where $I_{1}$ and $I_{2}$ are in $\mathcal{L}(R)$. Then

$$
\theta_{I_{1}} \cap \theta_{I_{2}}=\left\{J \in \mathcal{P}(R): I_{1} \nsubseteq J \text { and } I_{2} \nsubseteq J\right\} .
$$

Since each $k$-ideal of $R$ is idempotent so $I_{1} I_{2}=I_{1} \cap I_{2}$. Thus $\theta_{I_{1}} \cap \theta_{I_{2}}=\theta_{I_{1} \cap I_{2}}$. So $\theta_{I_{1}} \cap \theta_{I_{2}}$ belongs to

$\mathfrak{I}(\mathcal{P}(R))$.

Let $\left\{\theta_{I_{i}}\right\}_{i \in \Sigma}$ be an arbitrary family of members of $\mathfrak{I}(\mathcal{P}(R))$. Then

$$
\begin{aligned}
\bigcup_{i \in \Omega} \theta_{I_{i}} & =\bigcup_{i \in \Omega}\left\{J \in \mathcal{P}(R): I_{i} \not \subseteq J\right\} \\
& =\left\{J \in \mathcal{P}(R): \exists l \in \Omega \text { so that } I_{l} \nsubseteq \subseteq J\right\}=\theta_{\sum_{i \in \Omega} I_{i}},
\end{aligned}
$$

where $\sum_{i \in \Omega} I_{i}$ is the $k$-ideal generated by $\bigcup_{i \in \Omega} I_{i}$.

Hence $\mathfrak{I}(\mathcal{P}(R))$ is a topology on $\mathcal{P}(R)$.

\subsection{Definition}

A fuzzy $k$-ideal $\mu$ of a hemiring $R$ is said to be normal if there exists $x \in R$ such that $\mu(x)=1$. If $\mu$ is a normal fuzzy $k$-ideal of $R$, then $\mu(0)=1$, hence $\mu$ is normal if and only if $\mu(0)=1$.

The proof of the following theorem is same as the proof of Theorem 4.4 of [29].

\subsection{Theorem}

A fuzzy subset $\lambda$ of a hemiring $R$ is a $k$-prime fuzzy $k$-ideal of $R$ if and only if

1) $\lambda^{0}=\{x \in R: \lambda(x)=\lambda(0)\}$ is a prime $k$-ideal of $R$.

2) $\operatorname{Im} \lambda=\{\lambda(x): x \in R\}$ contains exactly two elements.

3) $\lambda(0)=1$.

\subsection{Corollary}

Every $k$-prime fuzzy $k$-ideal of a hemiring is normal.
Let $R$ be a hemiring in which each fuzzy $k$-ideal is idempotent, $\mathcal{L}_{R}$ the lattice of fuzzy normal $k$-ideals of $R$ and $\mathcal{F P} P_{R}$ the set of all proper fuzzy $k$-prime $k$-ideals of $R$. For any fuzzy normal $k$-ideal $\lambda$ of $R$, we define $\theta_{\lambda}=\left\{\mu \in \mathcal{F} P_{R}: \lambda \pm \mu\right\}$ and $\tau\left(\mathcal{F P} P_{R}\right)=\left\{\lambda \in \mathcal{L}_{R}\right\}$.

A fuzzy $k$-ideal $\lambda$ of $R$ is called proper if $\lambda \neq \mathbb{R}$, where $\mathbb{R}$ is the fuzzy $k$-ideal of $R$ defined by $\mathbb{R}(x)=1$, $\forall x \in R$.

\subsection{Theorem}

The set $\tau\left(\mathcal{F} P_{R}\right)$ forms a topology on the set $\mathcal{F} P_{R}$.

Proof. 1) $\theta_{\Phi}=\left\{\mu \in \mathcal{F} P_{R}: \Phi \not \mid \mu\right\}=\phi$, where $\phi$ is the usual empty set and $\Phi$ is the characteristic function of $k$-ideal $\{0\}$. This follows since each $k$-prime fuzzy $k$-ideal of $R$ is normal. Thus the empty subset belongs to $\tau\left(\mathcal{F} P_{R}\right)$.

2) $\theta_{\mathbb{R}}=\left\{\mu \in \mathcal{F} P_{R}: \mathbb{R} \not \equiv \mu\right\}=\mathcal{F P} P_{R}$. This is true, since $\mathcal{F} P_{R}$ is the set of proper $k$-prime fuzzy $k$-ideals of $R$. So $\theta_{\mathbb{R}}=\mathcal{F} P_{R}$ is an element of $\tau\left(\mathcal{F} P_{R}\right)$.

3) Let $\theta_{\delta_{1}}, \theta_{\delta_{2}} \in \tau\left(\mathcal{F} P_{R}\right)$ with $\delta_{1}, \delta_{2} \in \mathcal{L}_{R}$.

Then $\theta_{\delta_{1}} \cap \theta_{\delta_{2}}=\left\{\mu \in \mathcal{F} P_{R}: \delta_{1} \not \equiv\right.$ and $\left.\delta_{2} \not \equiv \mu\right\}$. Since each fuzzy $k$-ideal of $R$ is idempotent, this implies $\delta_{1} \delta_{2}=\delta_{1} \wedge \delta_{2}$. Thus

$$
\theta_{\delta_{1}} \cap \theta_{\delta_{2}}=\left\{\mu \in \mathcal{F} P_{R}: \delta_{1} \not \equiv \mu \text { and } \delta_{2} \not \equiv \mu\right\}=\theta_{\delta_{1} \wedge \delta_{2}} .
$$

4) Let us consider an arbitrary family $\left\{\delta_{i}\right\}_{i \in I}$ of fuzzy $k$-ideals of $R$. Since

$$
\begin{aligned}
\bigcup_{i \in I} \theta_{\delta_{i}} & =\bigcup_{i \in I}\left\{\mu \in \mathcal{F} P_{R}: \delta_{i} \not \equiv \mu\right\} \\
& =\left\{\mu \in \mathcal{F} P_{R}: \exists^{\prime} s k \in I \text { so that } \delta_{k} \not \equiv \mu\right\} .
\end{aligned}
$$

Note that

$$
\begin{aligned}
\left(\sum_{i \in I} \delta_{i}\right)(x)= & \underset{x+a_{1}+a_{2}+\cdots=b_{1}+b_{2}+\cdots}{\vee}\left\{\delta_{1}\left(a_{1}\right) \wedge \delta_{2}\left(a_{2}\right) \wedge \cdots\right. \\
& \left.\wedge \delta_{1}\left(b_{1}\right) \wedge \delta_{2}\left(b_{2}\right) \wedge \cdots\right\}
\end{aligned}
$$

where $a_{1}, a_{2}, \cdots, b_{1}, b_{2}, \cdots \in R$ and only a finite number of the $a_{i}^{\prime} s$ and $b_{i}^{\prime} s$ are not zero. Since $\delta_{i}(0)=1$, therefore we are considering the infimum of a finite number of terms because $1^{\prime} s$ are effectively not being considered. Now, if for some $k \in I, \quad \delta_{k} \$ \mu$, then there exists $x \in R$ such that $\delta_{k}(x)>\mu(x)$. Consider the particular expression for $X$ in which $a_{k}=x, b_{k}=0$ and $a_{i}=b_{i}=0$ for all $i \neq k$. We see that $\delta_{k}(x)$ is an element of the set whose supremum is defined to be $\left(\sum_{i \in I} \delta_{i}\right)(x)$.

Thus $\left(\sum_{i \in I} \delta_{i}\right)(x) \geq \delta_{k}(x)>\mu(x)$. This implies $\left(\sum_{i \in I} \delta_{i}\right)(x)>\mu(x)$ that is $\sum_{i \in I} \delta_{i} \not \equiv \mu$.

Hence $\delta_{k} \not \equiv \mu$ for some $k \in I$ implies $\sum_{i \in I} \delta_{i} \not \equiv \mu$. 
Conversely, suppose that $\sum_{i \in I} \delta_{i} \not \mu$ then there exists an element $x \in R$ such that $\left(\sum_{i \in I} \delta_{i}\right)(x)>\mu(x)$.

This means that

$$
\begin{aligned}
& \underset{x+a_{1}+a_{2}+\cdots=b_{1}+b_{2}+\cdots}{\vee}\left\{\delta_{1}\left(a_{1}\right) \wedge \delta_{2}\left(a_{2}\right) \wedge \cdots\right. \\
& \left.\wedge \delta_{1}\left(b_{1}\right) \wedge \delta_{2}\left(b_{2}\right) \wedge \cdots\right\}>\mu(x) .
\end{aligned}
$$

Now, if all the elements of the set (whose supremum we are taking) are individually less than are equal to $\mu(x)$, then we have

$$
\begin{aligned}
\left(\sum_{i \in I} \delta_{i}\right)(x)= & \underset{x+a_{1}+a_{2}+\cdots=b_{1}+b_{2}+\cdots}{\vee}\left\{\delta_{1}\left(a_{1}\right) \wedge \delta_{2}\left(a_{2}\right) \wedge \cdots\right. \\
& \left.\wedge \delta_{1}\left(b_{1}\right) \wedge \delta_{2}\left(b_{2}\right) \wedge \cdots\right\} \\
\leq & \mu(x)
\end{aligned}
$$

which does not agree with what we have assumed. Thus, there is at least one element of the set (whose supremum we are taking), say,

$$
\delta_{1}\left(a_{1}^{\prime}\right) \wedge \delta_{2}\left(a_{2}^{\prime}\right) \wedge \cdots \wedge \delta_{1}\left(b_{1}^{\prime}\right) \wedge \delta_{2}\left(b_{2}^{\prime}\right) \wedge \cdots>\mu(x) .
$$

$\left(x+a_{1}^{\prime}+a_{2}^{\prime}+\cdots=b_{1}^{\prime}+b_{2}^{\prime}+\cdots\right.$ being the corresponding breakup of $x$, where only a finite number of $a_{i}^{\prime} s$ and $b_{i}^{\prime} s$ are not zero).

Thus,

$$
\begin{aligned}
& \delta_{1}\left(a_{1}^{\prime}\right) \wedge \delta_{2}\left(a_{2}^{\prime}\right) \wedge \cdots \wedge \delta_{1}\left(b_{1}^{\prime}\right) \wedge \delta_{2}\left(b_{2}^{\prime}\right) \wedge \cdots>\mu(x) \\
& \geq \mu_{1}\left(a_{1}^{\prime}\right) \wedge \mu_{2}\left(a_{2}^{\prime}\right) \wedge \cdots \wedge \mu_{1}\left(b_{1}^{\prime}\right) \wedge \mu_{2}\left(b_{2}^{\prime}\right) \wedge \cdots
\end{aligned}
$$

Let

$$
\delta_{1}\left(a_{1}^{\prime}\right) \wedge \delta_{2}\left(a_{2}^{\prime}\right) \wedge \cdots \wedge \delta_{1}\left(b_{1}^{\prime}\right) \wedge \delta_{2}\left(b_{2}^{\prime}\right) \wedge \cdots=\delta_{p}\left(x_{p}^{\prime}\right)
$$

and

$$
\mu_{1}\left(a_{1}^{\prime}\right) \wedge \mu_{2}\left(a_{2}^{\prime}\right) \wedge \cdots \wedge \mu_{1}\left(b_{1}^{\prime}\right) \wedge \mu_{2}\left(b_{2}^{\prime}\right) \wedge \cdots=\mu_{p}\left(x_{p}^{\prime}\right)
$$

where $p \in I$.

So, $\delta_{p}\left(x_{p}^{\prime}\right) \succ \mu_{p}\left(x_{p}^{\prime}\right)$ it follows that $\delta_{p} \not \equiv \mu$ for some $p \in I$.

Hence $\sum_{i \in I} \delta_{i} \not \equiv \mu$ implies that $\delta_{p} \not \equiv \mu$ for some $p \in I$.

Hence the two statements 1) $\sum_{i \in I} \delta_{i} \not \equiv \mu$ and 2)

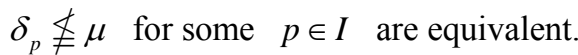

Hence

$$
\begin{aligned}
\bigcup_{i \in I} \theta_{\delta_{i}} & =\bigcup_{i \in I}\left\{\mu \in P_{R}: \delta_{i} \not \equiv \mu\right\} \\
& =\bigcup_{i \in I}\left\{\mu \in P_{R}: \sum_{i \in I} \delta_{i} \not \equiv \mu\right\}=\theta_{\sum_{i \in I} \theta_{i}}
\end{aligned}
$$

because, $\sum_{i \in I} \delta_{i}$ is also a fuzzy $k$-ideal of $R$.

Thus, $\bigcup_{i \in I} \theta_{\delta_{i}} \in \tau\left(P_{R}\right)$. Hence it follows that $\tau\left(P_{R}\right)$ forms a topology on the set $P_{R}$.

\section{Conclusion}

In the study of fuzzy algebraic system, the fuzzy ideals with special properties always play an important role. In this paper we study those hemirings for which each fuzzy $k$-ideal is idempotent. We characterize these hemirings in terms of prime and semiprime fuzzy $k$-ideals. In the future we want to study those hemirings for which each fuzzy one sided $k$-ideal is idempotent and also those hemirings for which each fuzzy $k$-bi-ideal is idempotent.

\section{REFERENCES}

[1] H. S. Vandiver, "Note on a Simple Type of Algebra in Which Cancellation Law of Addition Does Not Hold," Bulletin of the American Mathematical Society, Vol. 40, No. 12, 1934, pp. 914-920. doi:10.1090/S0002-9904-1934-06003-8

[2] A. W. Aho and J. D. Ullman, "Introduction to Automata Theory, Languages and Computation," Addison Wesley, Reading, 1976.

[3] D. B. Benson, "Bialgebras: Some Foundations for Distributed and Concurrent Computation," Fundamenta Informatica, Vol. 12, 1989, pp. 427-486.

[4] J. H. Conway, "Regular Algebra and Finite Machines," Chapman and Hall, London, 1971.

[5] K. Glazek, "A Guide to Literature on Semirings and Their Applications in Mathematics and Information Sciences with Complete Bibliography," Kluwer Academic Publishers, Berlin, 2002.

[6] J. S. Golan, "Semirings and Their Applications," Kluwer Academic Publishers, Berlin, 1999. doi:10.1007/978-94-015-9333-5

[7] U. Hebisch and H. J. Weinert, "Semirings: Algebraic Theory and Applications in the Computer Science," World Scientific, Singapore, 1998. doi:10.1142/3903

[8] W. Kuich and A. Salomma, "Semirings, Automata, Languages," Springer Verlag, Berlin, 1986. doi:10.1007/978-3-642-69959-7

[9] S. Eilenberg, "Automata, Languages and Machines," Academic Press, New York, 1974.

[10] E. T. Lee and L. A. Zadeh, "Note on Fuzzy Languages," Information Sciences, Vol. 1, No. 4, 1969, pp. 421-434. doi:10.1016/0020-0255(69)90025-5

[11] M. Henriksen, "Ideals in Semirings with Commutative Addition," Notices of the American Mathematical Society, Vol. 6, 1958, p. 321.

[12] K. Iizuka, "On the Jacobson Radial of a Semiring," Tohoku Mathematical Journal, Vol. 11, 1959, pp. 409-421.

[13] D. R. LaTorre, "On $h$-Ideals and $k$-Ideals in Hemirings," Publicationes Mathematicae (Debrecen), Vol. 12, 1965, pp. 219-226.

[14] L. A. Zadeh, "Fuzzy Sets," Infection Control, Vol. 8, No. 3, 1965, pp. 338-353. doi:10.1016/S0019-9958(65)90241-X

[15] J. Ahsan, K. Saifullah and M. Farid Khan, "Fuzzy Semir- 
ings," Fuzzy Sets Systems, Vol. 60, No. 3, 1993, pp. 309320. doi:10.1016/0165-0114(93)90441-J

[16] J. Ahsan, "Semirings Characterized by Their Fuzzy Ideals," Journal of Fuzzy Mathematics, Vol. 6, 1998, pp. 181-192.

[17] M. Akram and W. A. Dudek, "Intuitionistic Fuzzy Left k-Ideals of Semirings," Soft Computing, Vol. 12, No. 9, 2008, pp. 881-890. doi:10.1007/s00500-007-0256-x

[18] S. I. Baik and H. S. Kim, "On Fuzzy k-Ideals in Semirings," Kangweon Kyungki Mathematical Journal, Vol. 8, No. 2, 2000, pp. 147-154.

[19] T. K. Dutta and B. K. Biswan, "Fuzzy k-Ideals of Semirings," Bulletin of Calcutta Mathematical Society, Vol. 87, 1995, pp. 91-96.

[20] S. Ghosh, "Fuzzy k-Ideals of Semirings," Fuzzy Sets Systems, Vol. 95, No. 1, 1998, pp. 103-108. doi:10.1016/S0165-0114(96)00306-5

[21] C. B. Kim and M. Park, "k-Fuzzy Ideals in Semirings," Fuzzy Sets Systems, Vol. 81, No. 2, 1996, pp. 281-286. doi:10.1016/0165-0114(95)00161-1

[22] J. Zhan and Z. Tan, "T-fuzzy $k$-Ideals of Semirings," Scientiae Mathematicae Japonica, Vol. 58, 2003, pp. 597601.

[23] W. A. Dudek, M. Shabir and R. Anjum, "Characterizations of Hemirings by Their h-Ideals," Computers \& Mathematics with Applications, Vol. 59, No. 9, 2010, pp. 3167-3179. doi:10.1016/j.camwa.2010.03.003
[24] X. Ma and J. Zhan, "On Fuzzy h-Ideals of Hemirings," Journal of Systems Science and Complexity, Vol. 20, No. 3, 2007, pp. 470-478. doi:10.1007/s11424-007-9043-0

[25] X. Ma and J. Zhan, "Generalized Fuzzy $h$-Bi-Ideals and h-Quasi-Ideals of Hemirings," Information Sciences, Vol. 179, No. 9, 2009, pp. 1249-1268. doi:10.1016/j.ins.2008.12.014

[26] Y. Yin, X. Huang, D. Xu and F. Li, "The Characterization of h-Semisimple Hemirings," International Journal of Fuzzy Systems, Vol. 11, No. 2, 2009, pp. 116-122.

[27] Y. Yin and H. Li, "The Characterizations of $h$-Hemiregular Hemirings and $h$-Intra-Hemiregular Hemirings," Information Sciences, Vol. 178, No. 17, 2008, pp. 34513464. doi:10.1016/i.ins.2008.04.002

[28] J. Zhan, "On Properties of Fuzzy Left $h$-Ideals in Hemirings with t-Norms," International Journal of Mathematics and Mathematical Sciences, No. 19, 2005, pp. 3127-3144. doi:10.1155/IJMMS.2005.3127

[29] J. Zhan and W. A. Dudek, "Fuzzy h-Ideals of Hemirings," Information Sciences, Vol. 177, No. 3, 2007, pp. 876-886. doi:10.1016/j.ins.2006.04.005

[30] M. K. Sen and P. Mukhopadhyay, "von Neumann Regularity in Semirings," Kyungpook Mathematical Journal, Vol. 35, 1995, pp. 249-258.

[31] G. Birkhoff, "Lattice Theory," American Mathematical Society, Providence, 1954. 